\title{
CHCHD2 and CHCHD10 regulate mitochondrial dynamics and integrated stress response
}

\author{
Yu Ruan ${ }^{1,2,3}$, Jiaqiao Hu${ }^{1,2}$, Yaping Che $e^{1,2}$, Yanyan Liu ${ }^{1,2}$, Zhenhuan Luo ${ }^{1,2}$, Jin Cheng ${ }^{3}$, Qi Han ${ }^{3,4}$, He He $\mathbb{D}^{5 凶}$ and Qinghua Zhou $\mathbb{D}^{1,2}$
}

(c) The Author(s) 2022

Mitochondrial dysfunction is becoming one of the main pathology factors involved in the etiology of neurological disorders. Recently, mutations of the coiled-coil-helix-coiled-coil-helix domain containing 2 (CHCHD2) and 10 (CHCHD10) which encode two homologous proteins that belong to the mitochondrial $\mathrm{CHCH}$ domain protein family, are linked to Parkinson's disease and amyotrophic lateral sclerosis (ALS)/frontotemporal dementia (FTD), respectively. However, the physiological and pathological roles of these twin proteins have not been well elaborated. Here, we show that, in physiological conditions, CHCHD2 and CHCHD10 interact with OMA1 and suppress its enzyme activity, which not only restrains the initiation of the mitochondrial integrated response stress (mtlSR), but also suppresses the processing of OPA 1 for mitochondrial fusion. Further, during mitochondria stress-induced by carbonyl cyanide m-chlorophenylhydrazone (CCCP) treatment, CHCHD2 and CHCHD10 translocate to the cytosol and interacte with elF2a, which attenuates mtISR overactivation by suppressing elF2a phosphorylation and its downstream response. As such, knockdown of CHCHD2 and CHCHD10 triggers mitochondrial ISR, and such cellular response is enhanced by CCCP treatment. Therefore, our findings demonstrate the first " $\mathrm{mtISR}$ suppressor" localized in mitochondria for regulating stress responses in mammalian cells, which has a profound pathological impact on the $\mathrm{CHCH} 2 / \mathrm{CHCH} 10$-linked neurodegenerative disorder.

Cell Death and Disease (2022)13:156; https://doi.org/10.1038/s41419-022-04602-5

\section{INTRODUCTION}

Mitochondria are double membrane-bound subcellular organelles well-known for producing ATP and controlling metabolism. Mitochondria are highly dynamic organelles that undergo continual cycles of fusion and fission to maintain their shape, structure, and function. Fusion of mitochondrial outer (OM) and inner membranes (IM) are mediated by mitofusins (MFN1 and MFN2) and optic atrophy 1 (OPA1), respectively [1]. Mitochondrial fission is executed by dynamin-related protein 1(Drp1) which is recruited from the cytosol to mitochondria by mitochondrial outer membrane protein Fis1, Mff4, Mid49, Mid51 [2]. OPA1, a dynamin-like GTPase which is processed by two mitochondrial proteases OMA1 and Yme1L after entering into mitochondria, plays a role in remodeling cristae and the release of cytochrome $c$ during apoptosis in addition to mediating mitochondrial inner membrane fusion [3-6]. Defective mitochondrial dynamic leads to mitochondrial dysfunction and are relevant with neurodegenerative disease [7-9]. Mitochondrial dysfunction will trigger an integrated stress response that endures periods of stress. Under stress, mitochondrial protease OMA1 will cleavage DELE1 into a short form that translocates from mitochondria to cytosol, where it binds to and activates the protein kinase HRI that phosphorylates elF2a. Phosphorylated elF2a enhances the expression of ATF4 which promotes translation of a wide range of adaptive genes [10-12].
In recent years, mutations at two mitochondrial homologous proteins $\mathrm{CHCHD} 2$ and $\mathrm{CHCHD} 10$ have been identified as a wild spectrum of neurodegeneration disease. Mutations of $\mathrm{CHCHD} 2$ are mainly linked to Parkinson's disease [13]. Mutations of CHCHD10 are associated with ALS- and FTD-like symptoms [14-18], Charcot-Marie-Tooth disease type 2 (CMT2) $[19,20]$, spinal motor neuronopathy [21], motor neuron disease [15], and mitochondrial myopathy [22]. CHCHD2 and CHCHD10 belong to the mitochondrial coiled-coil-helix-coiled-coil-helix $(\mathrm{CHCH})$ domain protein family whose members are nucleus-encoded mitochondrial small proteins containing twin CX9C motifs ((CX9C)2) characterized by two cysteine residues separated by nine amino acids and are imported to the mitochondrial intermembrane space [23].

CHCHD2 and CHCHD10 form a $\sim 220 \mathrm{kDa}$ complex in the mitochondrial intermembrane space and cooperate to regulate mitochondrial function [24-26]. CHCHD2 has been reported to regulate oxidative phosphorylation (OXPHOS), promoting the expression of the COX4I2 subunit during stress [27, 28], inhibiting apoptosis [29]. Recently, it has been reported that CHCHD2 KO mice exhibited p62 inclusion formation and dopaminergic neuronal loss in an age-dependent manner [30].

CHCHD10 also regulates mitochondrial COX activity and mitochondrial respiration at hypoxia [31]. Many studies reported that $\mathrm{CHCHD} 2$ and $\mathrm{CHCHD} 10$ could play a role in mitochondrial dynamics and cristae organization. Disease-associated mutation of

\footnotetext{
${ }^{1}$ The Sixth Affiliated Hospital of Jinan University, Jinan University, Dongguan, Guangdong 523560, China. ${ }^{2}$ The Biomedical Translational Research Institute, Faculty of Medical Science, Jinan University, Guangzhou, Guangdong 510632, China. ${ }^{3}$ Department of Anatomy and Physiology, Shanghai Jiao Tong University School of Medicine, Shanghai 200025 , China. ${ }^{4}$ Shanghai Center for Brain Science and Brain-Inspired Technology, Shanghai 201210, China. ${ }^{5}$ Department of Anesthesiology, Renmin Hospital of Wuhan University, Wuhan, Hubei 430060, China. ${ }^{\bowtie}$ email: hehe2013@whu.edu.cn; gene@email.jnu.edu.cn Edited by Professor Massimiliano Agostini
}

Received: 13 May 2021 Revised: 12 January 2022 Accepted: 27 January 2022 Published online: 16 February 2022 
CHCHD2 and CHCHD10 has been found to lead to defects of mitochondrial dynamics and cristae.

At first, CHCHD2 and CHCHD10 were believed to be part of the mitochondrial contact site and cristae organizing system (MICOS) complex, which is crucial for mitochondrial membrane architecture and cristae organization, because they were observed enriched at cristae junctions, and their neurodegeneration associated mutations led to cristae abnormalities [14, 32-34]. However, the recent study would debate this view and suggest that neurodegenerative-disease-linked mutation of CHCHD10 or loss of $\mathrm{CHCHD} 2$ and $\mathrm{CHCHD} 10$ led to cristae abnormalities because of excessive processing of OPA1, while CHCHD2 or CHCHD10 single knockout did not lead to while CHCHD2 or CHCHD10 single knockout did not induce such effect $[35,36]$. In MEF cells, both mutation CHCHD10-S59L and simultaneous loss of $\mathrm{CHCHD} 2$ and $\mathrm{CHCHD} 10$ were also found to cause mitochondrial integrated stress response (mtISR) $[35,36]$, however, the mechanism is unknown. In this study, we have investigated the mechanism that $\mathrm{CHCHD} 2$ and $\mathrm{CHCHD} 10$ regulate mitochondrial dynamics and mtISR. We show that a single loss of $\mathrm{CHCHD} 2$ could result in OPA1 processing and abnormalities of cristae. We propose that $\mathrm{CHCHD} 2$ and $\mathrm{CHCHD} 10$ bind OMA1 to inhibit its activity under normal conditions. We also observe that $\mathrm{CHCHD} 2$ and $\mathrm{CHCHD} 10$ translocate from mitochondria to cytosol resulting in the interaction with elF2a, hence inhibiting elF2a phosphorylation and mtISR under stress conditions.

\section{CHCHD2 and CHCHD10 regulate mitochondrial dynamics and} ultrastructure

To validate whether CHCHD2 and CHCHD10 have the function of maintaining mitochondrial morphology in human cells, we knocked down CHCHD2 or/and CHCHD10 in Hela cells by shRNA (Fig. 1A). We found that CHCHD2 or/and CHCHD10 knockdown cells showed similar mitochondrial morphology with the control cells (Fig. 1B, C). However, when we further assessed the activities of mitochondrial fusion and fission via a photoactivatable GFP (PA-GFP) assay $[4,37]$, we found that the rate of mitochondrial fusion and fission was significantly attenuated in $\mathrm{CHCHD} 2$ single knockdown as well as $\mathrm{CHCHD} 2$ and $\mathrm{CHCHD} 10$ double knockdown cells compared with control cells (Fig. 1D and Supplementary Movie 1-4). Notably, the alteration of slow mitochondrial fusion and fission was not due to the change of cell viability after CHCHD2/10 knockdown (Supplementary Fig. 1). To study the molecular mechanism of alternations in mitochondrial dynamics upon the loss of $\mathrm{CHCHD} 2$ and $\mathrm{CHCHD} 10$, we detected expression levels of mitochondrial proteins involved in mitochondrial fusion-fission process by Western blot. In CHCHD2 single knockdown Hela cells, long forms of OPA1 (L-OPA1) decreased significantly while short forms of OPA1 (S-OPA1) processed by OMA1 increased in comparison with control cells (Fig. 1A). Interestingly, $\mathrm{CHCHD} 10$ single knockdown did not alter the processing of OPA1, however, CHCHD2 and CHCHD10 double knockdown further increased the cleavage of L-OPA1. Fis1 and phosphorylated DRP1 (Ser616), both of which promote mitochondrial fission, were dramatically reduced in CHCHD2 knockdown and $\mathrm{CHCHD} 2 / \mathrm{CHCHD} 10$ double knockdown cells. Other mitochondrial dynamics-related proteins, including Mfn1, Mfn2 and Mff had very similar expression levels between control cells and $\mathrm{CHCHD} 2 / \mathrm{CHCHD} 10$ knockdown cells (Fig. 1A). Hence, these results demonstrated that despite no effect on mitochondrial morphology, loss of CHCHD2/CHCHD10 slowed down the rate of mitochondrial fusion and fission.

Since OPA1 also plays a role in mitochondrial cristae organization [38], reduction of L-OPA1 in response to $\mathrm{CHCHD} 2 / \mathrm{CHCHD} 10$ knockdown also lead us to investigate whether $\mathrm{CHCHD2}$ and CHCHD10 regulated mitochondrial ultrastructure in Hela cells. Utilizing the transmission electron microscopy (TEM), we observed that $\mathrm{CHCHD} 10$ single knockdown cell lines showed normal cristae structure while $\mathrm{CHCHD} 2$ single knockdown cells, as well as CHCHD2 and CHCHD10 double knockdown cells, exhibited cristae abnormalities (Fig. 1E, F). This result was consistent with the OPA1 processing resulting from $\mathrm{CHCHD} 2$ knockdown (Fig. 1A). Given $\mathrm{CHCHD} 2$ and $\mathrm{CHCHD} 10$ knockdown interrupted mitochondrial integrity, it prompted us to further investigate how such $\mathrm{CHCHD2}$ / 10 deficiency-altered mitochondrial dynamics and ultrastructure would affect mitochondrial function and ROS production. To do this, we assessed cellular respiration by oximetry. The basal and maximal oxygen consumption was decreased in $\mathrm{CHCHD} 2$ or/and CHCHD10 knockdown cells when compared with wild-type control cells (Supplementary Fig. 2A). Mitochondrial membrane potential measured by Tetramethylrhodamine methyl ester (TMRM) fluorescence was also reduced in comparison with wildtype control cells (Supplementary Fig. 2B). The ATP production was decreased in CHCHD2 and CHCHD10 knockdown cells related to wild-type control cells (Supplementary Fig. 2C). In addition, mitochondrial ROS production measured by the reagent MitoSOX increased significantly in Hela cells after CHCHD2 and CHCHD10 knockdown (Supplementary Fig. 2D). Together, our results demonstrate that $\mathrm{CHCHD} 2 / \mathrm{CHCHD} 10$ deficiency disrupts mitochondrial integrity, increases ROS production, and further compromises mitochondrial function in Hela cells.

\section{Loss of CHCHD2 and CHCHD10 induces mitochondrial ISR}

It has been previously reported that the abundance of $\mathrm{CHCHD} 2$ and CHCHD10 is increased after the loss of membrane potential [39]. To verify the report, we treated cells with carbonyl cyanide m-chlorophenylhydrazone (CCCP) and then detected CHCHD2 and $\mathrm{CHCHD} 10$ by Western blot assay. The results showd that CHCHD2 and CHCHD10 were robustly increased after CCCP treatment (Fig. 2A).

It has been also reported that $\mathrm{CHCHD} 2$ and $\mathrm{CHCHD} 10$ double knockout resulted in mitochondrial integrated stress response (ISR) instead of CHCHD2 or CHCHD10 single knockout in mouse embryonic fibroblasts at normal conditions [40]. CCCP treatment also triggers mitochondrial integrated stress response (ISR) [12]. To explore whether $\mathrm{CHCHD} 2$ and $\mathrm{CHCHD} 10$ can regulate ISR in human cells under normal conditions and stress conditions, we treated $\mathrm{CHCHD} 2$ or/and CHCHD10 knockdown cells with CCCP for $24 \mathrm{~h}$. Then, we determined the mRNA (ATF3, ATF4, DDIT3, CHAC1) and proteins (ASNS, PCK2, PSPH, p-elF2a) level of the ISR associated genes by RT-qPCR and Western blotting respectively (Fig. 2B, C). The ISR-related pathway proteins including ASNS, PCK2, and $p$-elF2 $a$ and the mRNA1 levels of ATF3, ATF4, DDIT3, and CHAC1 were slightly increased after $\mathrm{CHCHD} 2$ or $\mathrm{CHCHD} 10$ single knockdown, while the levels of proteins and mRNAs of these genes were increased significantly after $\mathrm{CHCHD} 2$ and $\mathrm{CHCHD} 10$ double knockdown under normal conditions (Fig. 2B, C), which was consistent with what has been reported in the mouse [41]. However, following CCCP treatment, CHCHD2 single knockdown could increase mRNA and protein levels of genes associated with the ISR pathway (Fig. 2B, C). Together, our results indicate that CHCHD2 and $\mathrm{CHCHD} 10$ double knockdown induce ISR under normal conditions, while CHCHD2 single knockdown could promote ISR under stress conditions.

\section{CHCHD2 and CHCHD10 interact with OMA1 to inhibit its protease activity}

As we knew, upon mitochondrial stress, DELE1 is cleaved by OMA1 in mitochondria and enters into the cytosol to interact with and assist HRI to phosphorylate the translation factor, elF2a $[10,11]$. To explore whether $\mathrm{CHCHD} 2$ and $\mathrm{CHCHD} 10$ regulated the cleavage of DELE1, we generated a Hela cell line stably expressing FLAGtagged DELE1 by a retrovirus. Utilizing the immunofluorescence staining, we observed that CHCHD2 and CHCHD10 double knockdown promoted DELE1-FLAG to be accumulated into cytosol (Fig. 3A, B). These findings indicate that loss of CHCHD2 

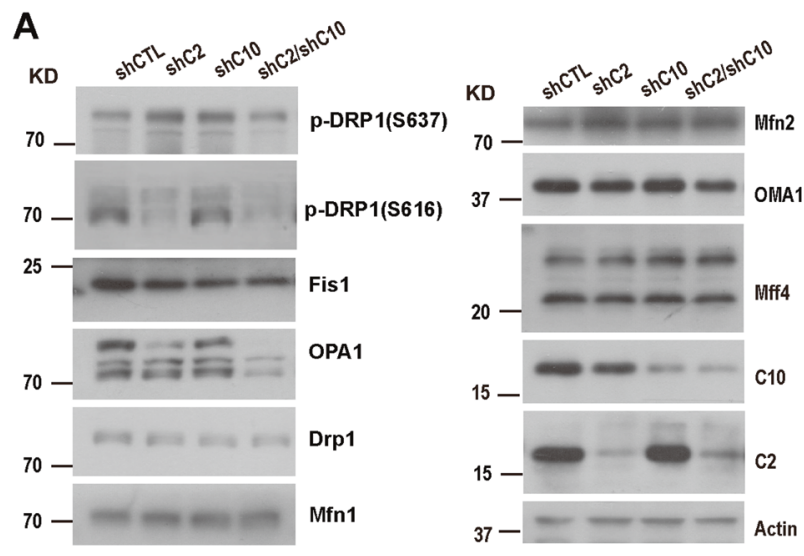

C

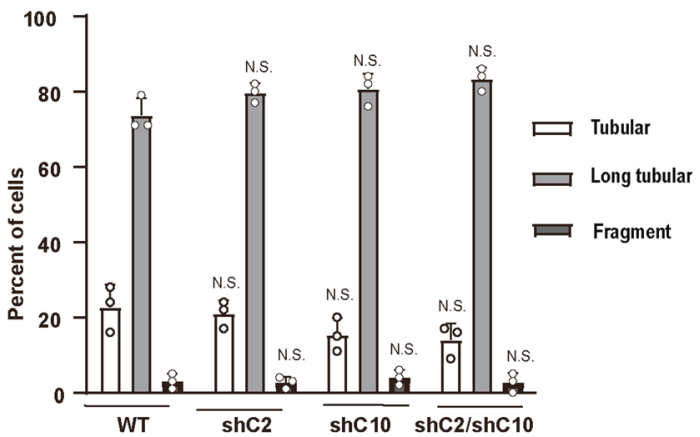

E

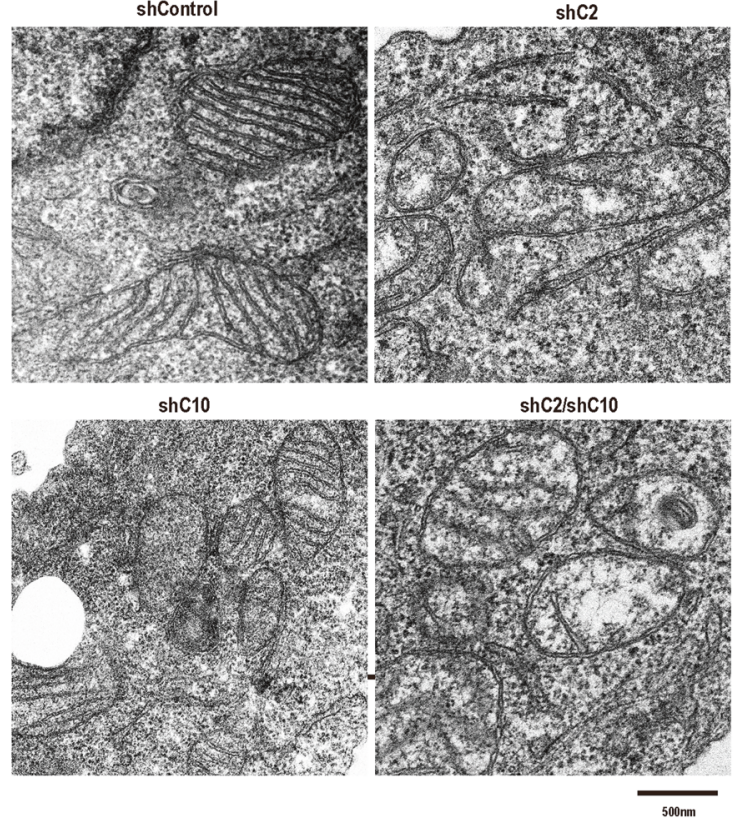

B
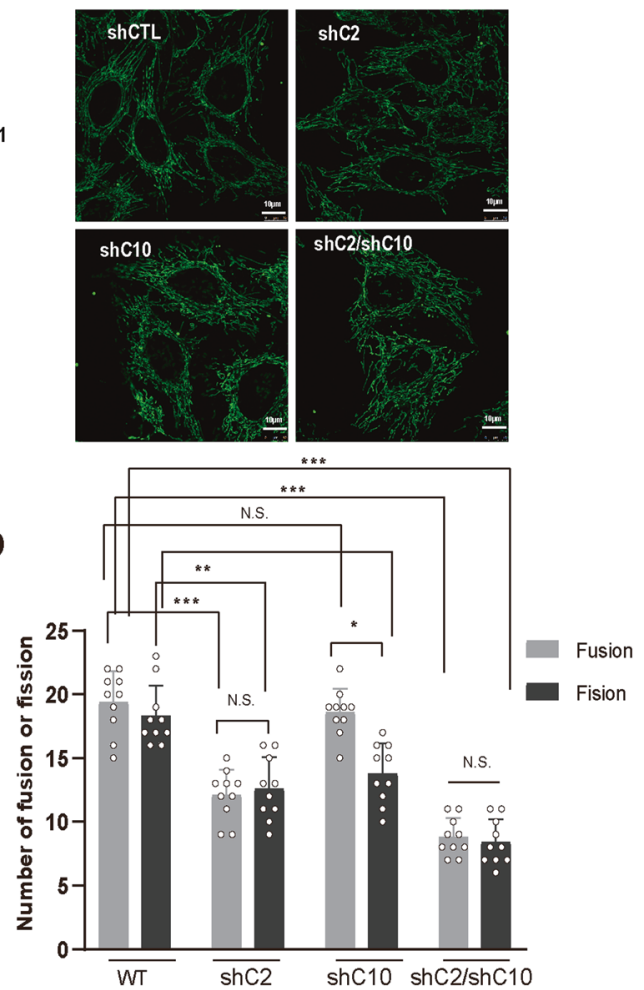

$\mathbf{F}$

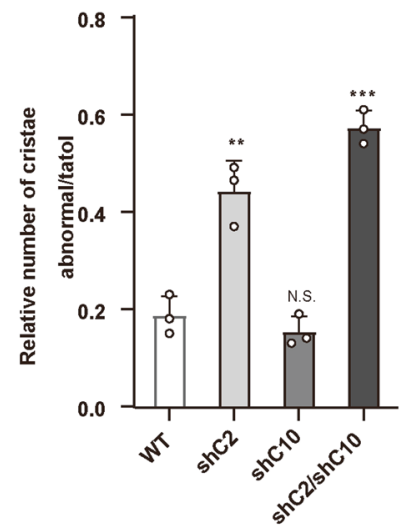

and $\mathrm{CHCHD} 10$ promotes the cleavage of DELE1 and triggers OMA1/DELE1-mediated ISR. We next investigate whether CHCHD10 and CHCHD2 would have a direct physical association with DELE1 to therefore suppress DELE1 cleavage. We, therefore, performed co-immunoprecipitation to examine whether DELE1 interacted with $\mathrm{CHCHD} 2$ and $\mathrm{CHCHD} 10$, and results showed that CHCHD10 and CHCHD2 failed to be precipitated by DELE1-FLAG under the normal conditions as well as following CCCP treatment, suggesting that $\mathrm{CHCHD} 2$ and $\mathrm{CHCHD} 10$ do not directly interact with DELE1 under both normal and stress conditions (Fig. 3C).

We also assessed whether OMA1 interacted with CHCHD2 and $\mathrm{CHCHD} 10$ by co-immunoprecipitation. We re-expressed
OMA1-WT and its protease-dead mutation E324Q in OMA1 KO Hct 116 cells. CHCHD2 and CHCHD10 were precipitated by OMA1FLAG and OMA1-E324Q-FLAG under normal conditions and CCCP treatment. CCCP increased the interaction OMA1 with CHCHD2 and CHCHD10. To our surprise, OMA1-WT interacted with $\mathrm{CHCHD} 2$ more strongly than its mutant OMA1-E324Q (Fig. 3D). We speculated that CHCHD2 and CHCHD10 could interact with the active site of the protease of OMA1 to suppress its protease activity.

To further validate that $\mathrm{CHCHD} 2$ and $\mathrm{CHCHD} 10$ interact with OMA1. We re-expressed CHCHD2 WT, and its PD-related mutants CHCHD2-T61I, CHCHD2-R145Q in CHCHD2 knockout cells 
Fig. 1 CHCHD2/CHCHD10 knockdown leads to a dynamic change of mitochondrial morphology and cristae abnormalities. A Whole-cell lysates of control, shCHCHD2, shCHCHD10, shCHCHD2/CHCHD10 HeLa cells were analyzed for indicated protein expression by immunoblot. $\beta$-Actin was used as a loading control. B Mitochondrial morphology in control, CHCHD2 knockdown, CHCHD10 knockdown, and CHCHD2/ CHCHD10 knockdown HeLa cells immunostained for Hsp60 (Green) was visualized by confocal microscope. C Mitochondrial morphology described in B was counted according to the criteria detailed in Experimental Procedures (mean \pm s.d. of $n=3$ independent biological samples; two-way ANOVA with Tukey's multiple comparisons correction, N.S. not significant). D Comparison of mitochondrial fusion and fission between control and shCHCHD2, shCHCHD10, shCHCHD2/CHCHD10 HeLa cells. Ten photoactivated mitochondria labeled with mitoPA-GFP were tracked by time-lapse microscopy for $20 \mathrm{~min}$, and the number of mitochondrial fission and fusion events within 20 min was counted. Three independent cells were analyzed, the number of mitochondrial fission and mitochondrial fusions within 20 min were counted. Bars represent means \pm S.D. of three independent experiments. Statistical significance analysis was used by two-way ANOVA with Sidak's multiple comparisons test, ${ }^{*} P<0.05,{ }^{*} P<0.01,{ }^{* * *} P<0.001, N$.S. not significant. E Mitochondrial ultrastructure in control, shCHCHD2, shCHCHD10, shCHCHD2/CHCHD10 HeLa cells was analyzed by transmission electron microscope (TEM). F The relative number of mitochondria with abnormal cristae (ratio of abnormal mitochondria to total mitochondria) in control, shCHCHD2, shCHCHD10, shCHCHD2/ CHCHD10 was counted. 100 mitochondria were measured from 20 cells. Statistical significance was assessed by one-way ANOVA with Dunnett's multiple comparisons test; error bars represent means \pm SD of three independent experiments; ${ }^{*} P<0.05,{ }^{* * P} P<0.01,{ }^{* * *} P<0.001, \mathrm{~N} . \mathrm{S}$., not significant.

respectively, and re-expressed FLAG-tagged CHCHD10 WT and ALS-related mutants CHCHD10-S59L and CHCHD10-G66V in CHCHD10 knockout cells respectively. In parallel, we treated those cells with CCCP for $12 \mathrm{~h}$. We performed co-immunoprecipitation experiments with CHCHD2 and FLAG antibodies respectively, followed by Western blot analysis. As reported previously, CHCHD2 and CHCHD10 interacted with P32, and CHCHD2 as well as $\mathrm{CHCHD} 10$ also interacted with the mitochondrial proteases OMA1 and YME1L (Fig. 3E, F). Since it has been reported that OMA1 is not required for basal turnover of $\mathrm{CHCHD} 2$ and $\mathrm{CHCHD} 10$ $[41,42]$ and loss of CHCHD2 and CHCHD10 lead to OPA1 processing and cleavage of DELE1, we hypothesized that CHCHD2 and $\mathrm{CHCHD} 10$ could regulate proteinase activity of $Y \mathrm{me} 1 \mathrm{~L}$ and OMA 1 by binding them. Additionally, elF2a, a cytoplasmic matrix protein, was also identified to interact with $\mathrm{CHCHD} 2$ and $\mathrm{CHCHD} 10$ and their mutations as well (Fig. 3E, F). Furthermore, CCCP promoted the interaction between elF2a and CHCHD2/ CHCHD10 (Fig. 3E, F).

\section{CHCHD2 and CHCHD10 translocate from mitochondria to the cytosol}

It has been reported that $\mathrm{CHCHD} 2$ and $\mathrm{CHCHD} 10$ are mitochondrial proteins. Given that $\mathrm{CHCHD} 2$ and $\mathrm{CHCHD} 10$ interact with elF2a which is a cytoplasmic protein, we hypothesized that CHCHD2 and CHCHD10 may localize in the cytosol as well. To validate this hypothesis, we isolated mitochondria, nucleus, and cytosol of Hela cells with or without CCCP treatment, followed by Western blot analysis. Results showed that in the absence of CCCP, $\mathrm{CHCHD} 2$ and $\mathrm{CHCHD} 10$ mainly co-fractionated with mitochondrial protein (Fig. 4A). Interestingly, by increasing the time period of CCCP treatment, an abundance of $\mathrm{CHCHD} 2$ and $\mathrm{CHCHD} 10$ was increased in the nuclear, mitochondria, and cytosol (Fig. 4A, B). To further assess whether $\mathrm{CHCHD} 2$ and $\mathrm{CHCHD} 10$ are translocated in cytosol under stress conditions, we expressed $\mathrm{CHCHD2}$ and its PDassociated mutants CHCHD2-T61I and CHCHD2-R145Q in CHCHD2 KO Hela cells respectively, as well as expressed CHCHD10-FLAG, CHCHD10-S59L-FLAG, and CHCHD10-G66V-FLAG in CHCHD10 KO Hela cells respectively, followed by treatment with or without CCCP (Fig. 4C, D). Immunofluorescence analysis revealed that signals for CHCHD2 and CHCHD10 were mainly colocalized with CYCS marking mitochondria in the absence of CCCP, suggesting their mitochondria localization at normal conditions. Signals for CHCHD10-S59L showed aggregation at mitochondria, consistent with previous report under normal conditions $[33,40]$. After $24 \mathrm{~h}$ of CCCP treatment, signals intensity for CHCHD2 and CHCHD10 and their mutations were enhanced and showed a diffuse pattern. Of special note, signals for CYCS marking mitochondria were disappeared in some cells because of mitophagy, however, signals intensity for CHCHD2, and CHCHD2-T61I and CHCHD2-R145Q, CHCHD10, CHCHD10-S59L-FLAG, CHCHD10-G66V-FLAG were increased at cytosol (Fig. 4E, F). Together, these results indicate that $\mathrm{CHCHD} 2$ and $\mathrm{CHCHD} 10$ are mainly localized at mitochondria at normal conditions, CCCP force translocation of $\mathrm{CHCHD} 2$ and CHCHD10 from mitochondria to cytosol and nucleus. Since simultaneous loss of $\mathrm{CHCHD} 2$ and $\mathrm{CHCHD} 10$ promotes phosphorylation of elF2a under normal conditions and single loss of $\mathrm{CHCHD} 2$ promotes phosphorylation of elF2 2 under CCCP treatment (Fig. 2C) and interaction of CHCHD2 and CHCHD10 with elF2 $\alpha$ is also increased after CCCP treatment (Fig. 3B, C). Thus, we speculated that $\mathrm{CHCHD} 2$ and $\mathrm{CHCHD} 10$ may suppress phosphorylation of elF2a by binding it under CCCP treatment. Hence, $\mathrm{CHCHD} 2$ and $\mathrm{CHCHD} 10$ regulate ISR by inhibiting protease activity of OMA under normal conditions and phosphorylation of elF2a under CCCP treatment.

\section{P32 regulates mitochondrial morphology and mtISR}

P32, a multifunctional chaperone protein predominantly localizing in mitochondria, is functionally important for the maintenance of mitochondrial function. However, the function of P32 in mitochondrial morphology has been debated [43-46]. Given that P32 interacts with $\mathrm{CHCHD} 2$ and $\mathrm{CHCHD} 10$ (Fig. 3C-E, Fig. 5A), we were also interested to validate whether $\mathrm{P} 32$ regulates mitochondrial morphology and ISR. To this end, we generated P32 KD, CHCHD2/ P32 DKD, CHCHD10/P32 DKD, and CHCHD2/CHCHD10/P32 triple KD (TKD) HeLa cells by lentivirus-mediated shRNA and checked the change of protein level of the mitochondrial dynamics related key proteins. The loss of P32 in Hela cells significantly led to a decrease of L-OPA1 and an increase of the S-OPA1 (Fig. 5B). Immunofluorescence analysis revealed that P32 knockdown led to remarkable mitochondrial fragmentation in about $70 \%$ of Hela cells, whereas wild-type (WT) Hela cells showed almost all tubular mitochondria (Fig. 5C, D).

To test whether P32 could regulate ISR, we treated WT or shP32 Hela cells with CCCP for $12 \mathrm{~h}$. P32 knockdown resulted in an increase of ISR proteins under both normal conditions and CCCP treatment (Fig. 5E), suggesting that loss of P32 promotes ISR under normal conditions and stress conditions. Consistent with OPA1 processing, P32 knockdown also resulted in mitochondrial cristae abnormalities (Fig. 5E). Together, these data suggest that P32 coordinates with $\mathrm{CHCHD} 2$ and $\mathrm{CHCHD} 10$ to regulate mitochondrial morphology and ISR.

\section{OMA1 and Yme1L are responsible for the degradation of} CHCHD2 and CHCHD10

Although previous studies reported that $\mathrm{CHCHD} 2$ and $\mathrm{CHCHD} 10$ could be degraded by OMA1, when cells are subject to mitochondrial stressor, Actinoin, OMA1 knockout could not entirely restore $\mathrm{CHDHD} 2$ and $\mathrm{CHCHD} 10$ degradation after Actinonin treatment, suggesting that, besides OMA1, there are other proteases responsible for degrading CHCHD2 and CHCHD10 [41]. 
A
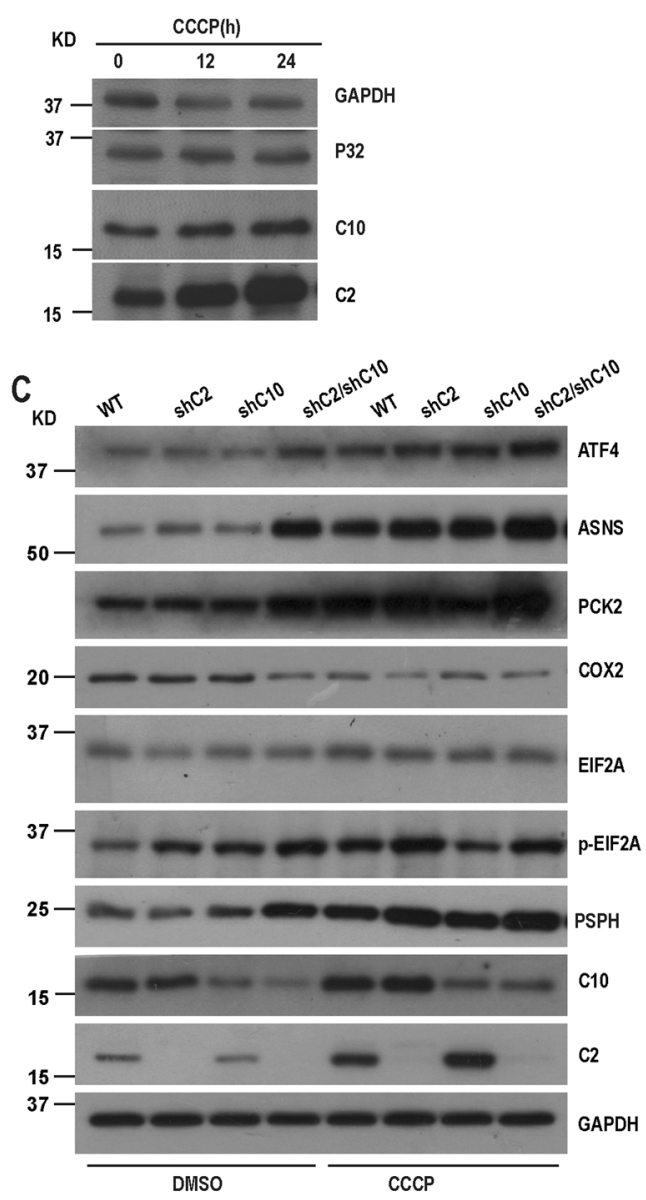

B
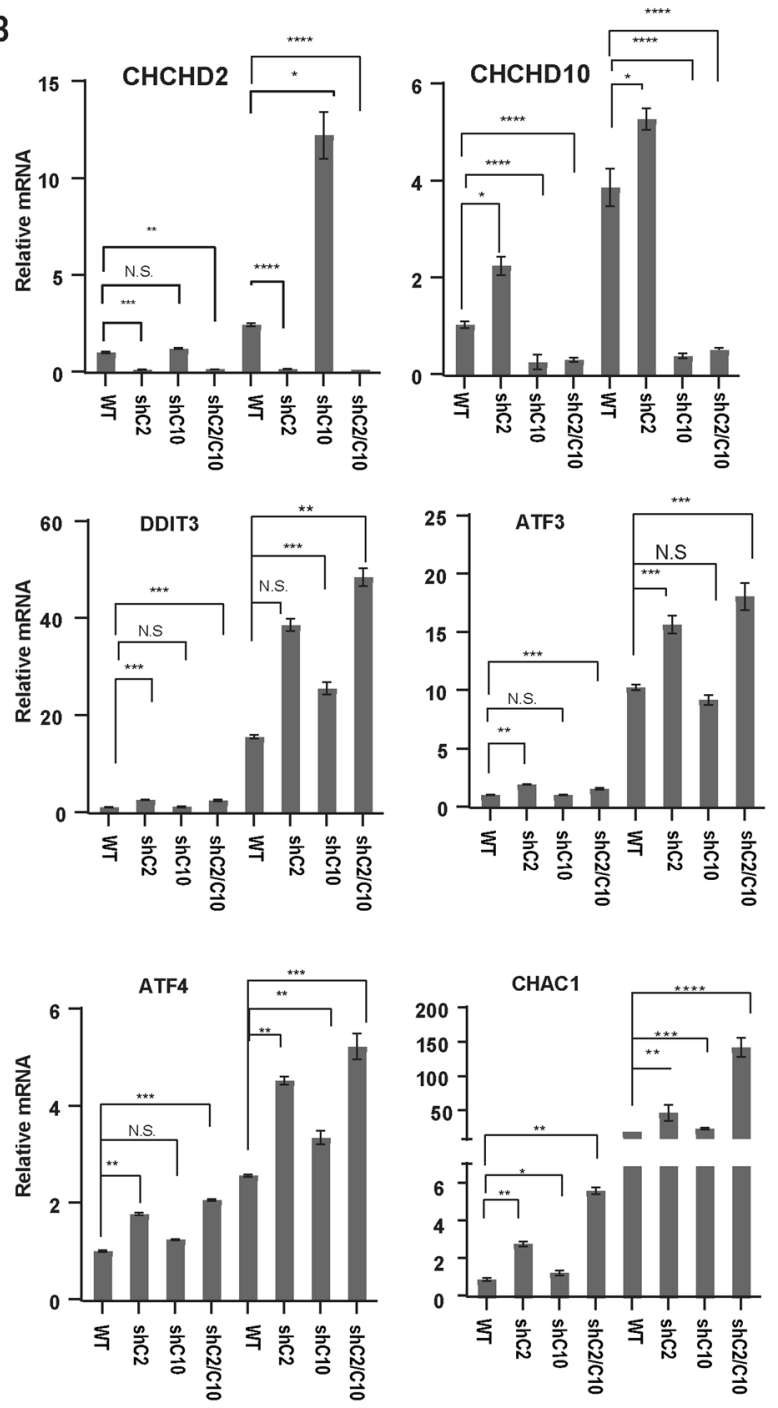

Fig. 2 Loss of CHCHD2 and CHCHD10 promotes mitochondrial integrated stress response. A Whole-cell lysates of HeLa cells treated with DMSO or CCCP $(10 \mathrm{mM}, 12 \mathrm{~h}$, or $24 \mathrm{~h})$ respectively were analyzed for indicated protein expression by immunoblot. GAPDH was used as a loading control. B HeLa cells were infected with control, shCHCHD2, shCHCHD10, shCHCHD2/CHCHD10 lentiviral particles respectively and cultured for 7 days, treated with CCCP $(10 \mathrm{mM})$ for $24 \mathrm{~h}$, and then relative transcript levels of the indicated gene was analyzed by RT-QPCR. Statistical significance analysis was used by two-way ANOVA with Sidak's multiple comparisons test, ${ }^{*} P<0.05,{ }^{* *} P<0.01,{ }^{* * *} P<0.001$, N.S. not significant. C HeLa cells were infected with control, shCHCHD2, shCHCHD10, shCHCHD2/CHCHD10 lentiviral particles respectively and cultured for 7 days, treated with CCCP $(10 \mathrm{mM})$ for $24 \mathrm{~h}$, and then the indicated protein expression was analyzed by immunoblot. GAPDH was used as a loading control.

Given mitochondrial protease OMA1 and Yme1L interact with CHCHD2 and CHCHD10 (Fig. 3D, E), we assess whether Yme1L is responsible for the degradation of $\mathrm{CHCHD} 2$ and $\mathrm{CHCHD} 10$. We generated OMA1 and Yme1L double knockout Hela cell lines with CRISPR-Cas9 system, and treated the cell lines with Oligomycin, a mitochondrial complex $\mathrm{V}$ inhibitor. Western Blot analysis showed that Oligomycin treatment caused $\mathrm{CHCHD} 2$ and $\mathrm{CHCHD} 10$ degradation, however, OMA1 and Yme1L knockout almost completely inhibited the degradation of CHCHD2 and CHCHD10 following oligomycin treatment (Fig. 6A). These data indicate that $\mathrm{Yme1L}$ is also required for stress-induced degradation of $\mathrm{CHCHD2}$ and $\mathrm{CHCHD} 10$.

Since CHCHD2 and CHCHD10 can be degraded under mitochondrial stress, suggesting that the degradation of $\mathrm{CHCHD} 2$ and $\mathrm{CHCHD} 10$ may play a critical role in the maintenance of cell function. We test whether the degradation rate of neurodegenerative disease-related $\mathrm{CHCHD} 2$ mutants would behave similarly to $\mathrm{CHCHD} 2$ in wildtype. To this end, we re-expressed wild-type
CHCHD2 and their mutants T61I and R145Q in CHCHD2 knockout cells and then treated these cell lines with Oligomycin in a timedependent manner. Western Blot analysis showed that the degradation rate of $\mathrm{CHCH} 2$ mutants, T61I and R145Q, was significantly slower than that of the wild-type CHCHD2 (Fig. 6B, $\mathrm{C})$, indicating that neurodegenerative disease-related $\mathrm{CHCHD2}$ mutants are resistant to degradation by Yme1L and OMA1 under mitochondrial stress.

\section{DISCUSSION}

CHCHD2 and CHCHD10 are homologous mitochondrial proteins and their mutations are identified with neurodegenerative disease [23]. Previous studies in mice have shown that CHCHD2 or CHCHD10 single knockout do not alter mitochondrial shape and ultrastructure, but $\mathrm{CHCHD} 2$ and $\mathrm{CHCHD} 10$ double knockout increase processing of and subsequent mitochondrial fragmentation and cristae abnormities [41]. 
A

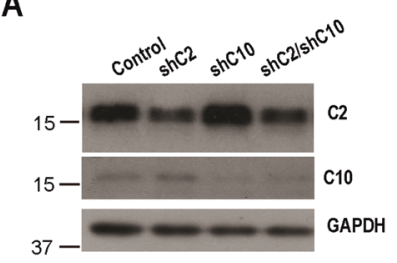

B
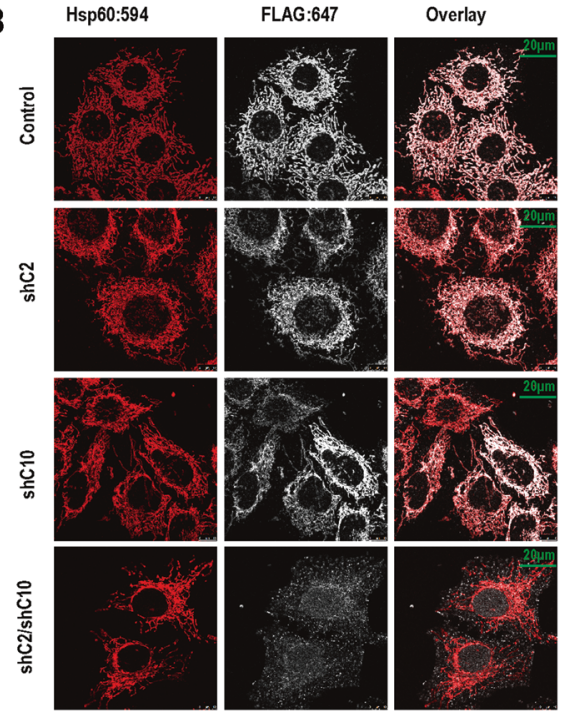

C

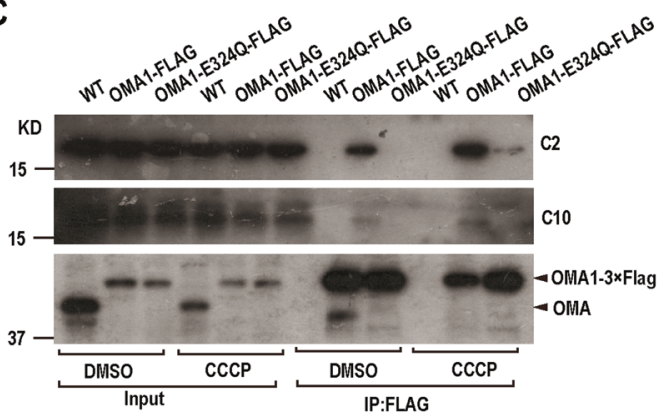

D

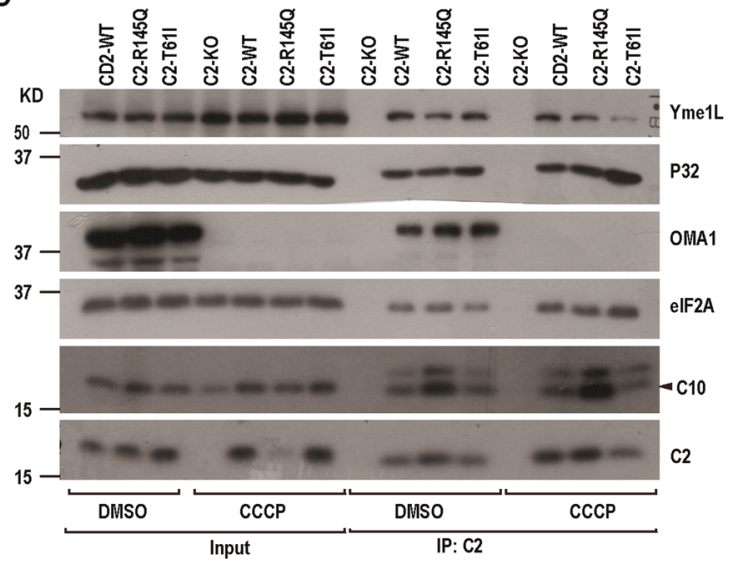

$\mathrm{E}$

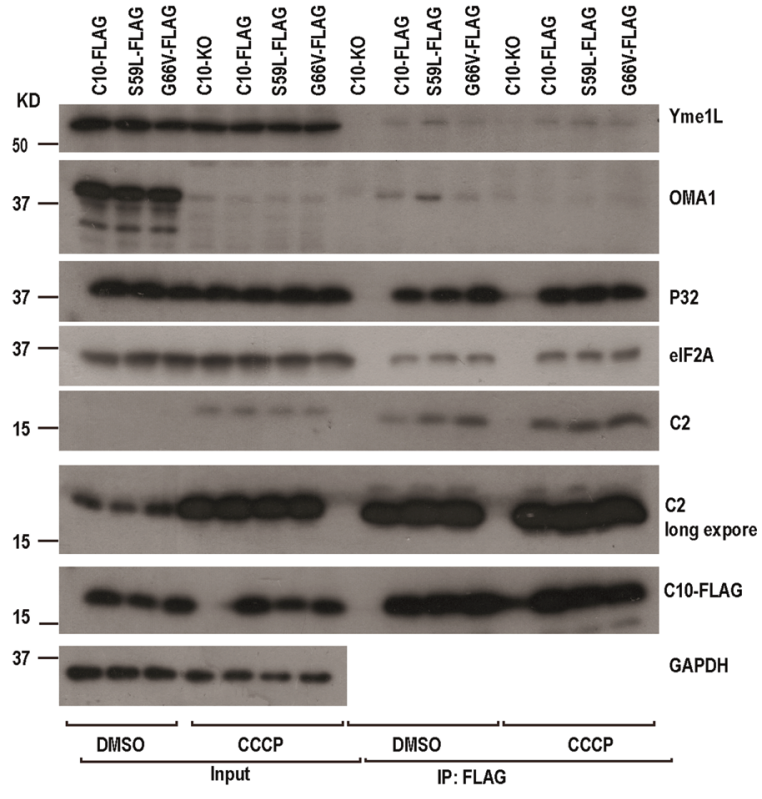

Fig. 3 CHCHD2 and CHCHD10 interact with OMA1. A DELE1-FLAG-expressing HeLa cells were infected with control or shCHCHD2, CHCHD10, $\mathrm{CHCHD} 2 / \mathrm{CHCHD} 10$ lentiviral particles and cultured for 5 days and then were treated with 10uM CCCP for $2 \mathrm{~h}$. Cell lysates were subjected to immunoblot using the indicated antibodies. B DELE1-FLAG-expressing HeLa cells were infected with control or shCHCHD2, CHCHD10, CHCHD2/CHCHD10 lentiviral particles and cultured for 5 days and were immunostained with Hsp60, FLAG, and localization of DELE1-FLAG (white) was visualized by confocal microscopy. C HCT116 and OMA1 KO HCT116 cells expressing FLAG-tagged Mus OMA1-WT or OMA1E324Q were treated with or without CCCP for $1 \mathrm{~h}$. Then cells were lysed and immunoprecipitated with anti-OMA1 antibody, and the protein samples were subjected to immunoblot using the indicated antibodies. D CHCHD2 KO Hela cells expressing either exogenous wild-type CHCHD2 or its mutation T61I or R145Q were treated with or without CCCP for $8 \mathrm{~h}$. Then cells were lysed and immunoprecipitated with anti$\mathrm{CHCHD} 2$, and the protein samples were subjected to immunoblot using the indicated antibodies. E CHCHD10 KO Hela cells expressing exogenous FLAG-tagged CHCHD10 or its mutation S59L or G66V were treated with or without CCCP for $8 \mathrm{~h}$. Then Cells were lysed and were immunoprecipitated with FLAG M2 resin, and the protein samples were subjected to immunoblot using the indicated antibodies.

In this study, we report that CHCHD2 single knockdown as well as $\mathrm{CHCHD} 2$ and $\mathrm{CHCHD} 10$ double knockdown can slow down mitochondrial fusion and fission (Fig. 1D). Because the rates of mitochondrial fusion and division are still similar, CHCHD2 or/and CHCHD10 knockdown cells show normal mitochondrial morphology. We found that, CHCHD2 knockdown, but not CHCHD10, caused OPA1 processing and disrupted mitochondrial cristae in HeLa cells, and CHCHD2 and CHCHD10 double knockdown was able to further such adverse effect (Fig. 1E, F).

Mitochondrial dysfunction could trigger mtISR. Simultaneous loss of $\mathrm{CHCHD} 2$ and $\mathrm{CHCHD} 10$ could leads to $\mathrm{mtISR}$ in mouse $[35,36]$. Under normal conditions, CHCHD2 and CHCHD10 single knockdown does not activate ISR, while CHCHD2 and CHCHD10 double knockdown leads to mtISR (Fig. 2B, C), which is consistent with previous reports [41]. However, we discovered that CHCHD2 single knockdown promotes mtISR triggered by CCCP treatment (Fig. 2B, C). Moreover, $\mathrm{CHCHD} 2$ and $\mathrm{CHCHD} 10$ are significantly increased after CCCP treatment (Fig. 2A), so we speculate that under normal conditions, the protein content of CHCHD2 or CHCHD10 is sufficient to inhibit mtISR, while under stress conditions, mitochondria damaged, the amount of CHCHD2 and CHCHD10 is insufficient to inhibit mtISR.

OMA1 maintains low activity under normal physiological conditions but can be activated to process OPA 1 to regulate 
A
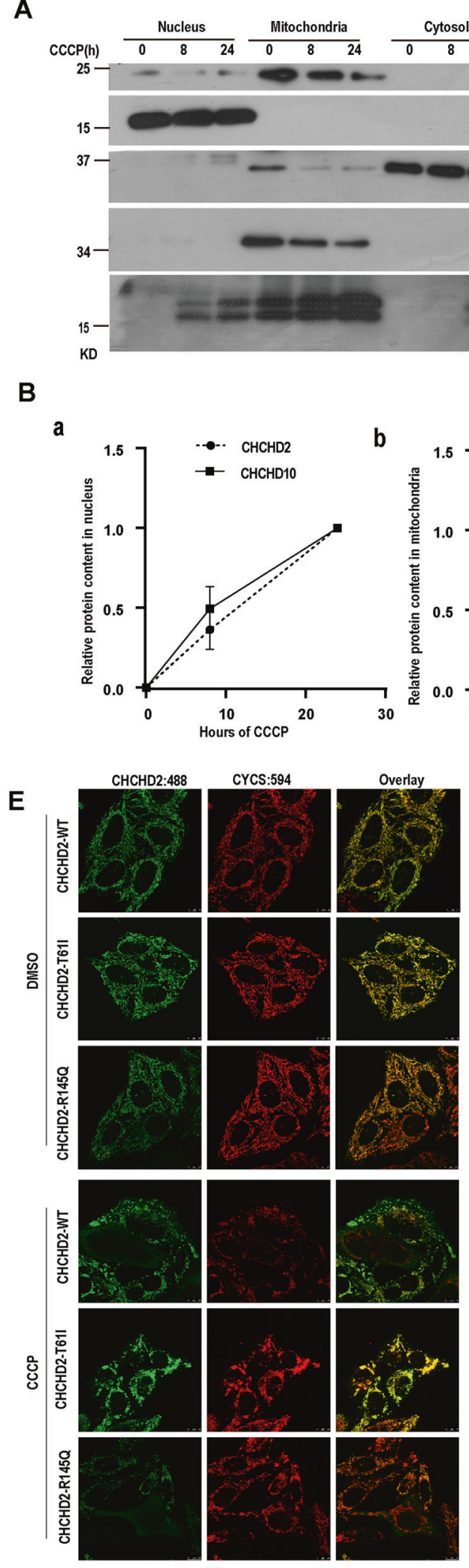

mitochondrial dynamics and cleave DELE1 to activate mtISR under stress conditions [47]. There are three possible reasons why $\mathrm{CHCHD} 2$ and $\mathrm{CHCHD} 10$ knockdown leads to the processing of OPA1 and DELE1: loss of CHCHD2 and CHCHD10 induce bioenergetic stress that activates OMA1; CHCHD2 and CHCHD10
C
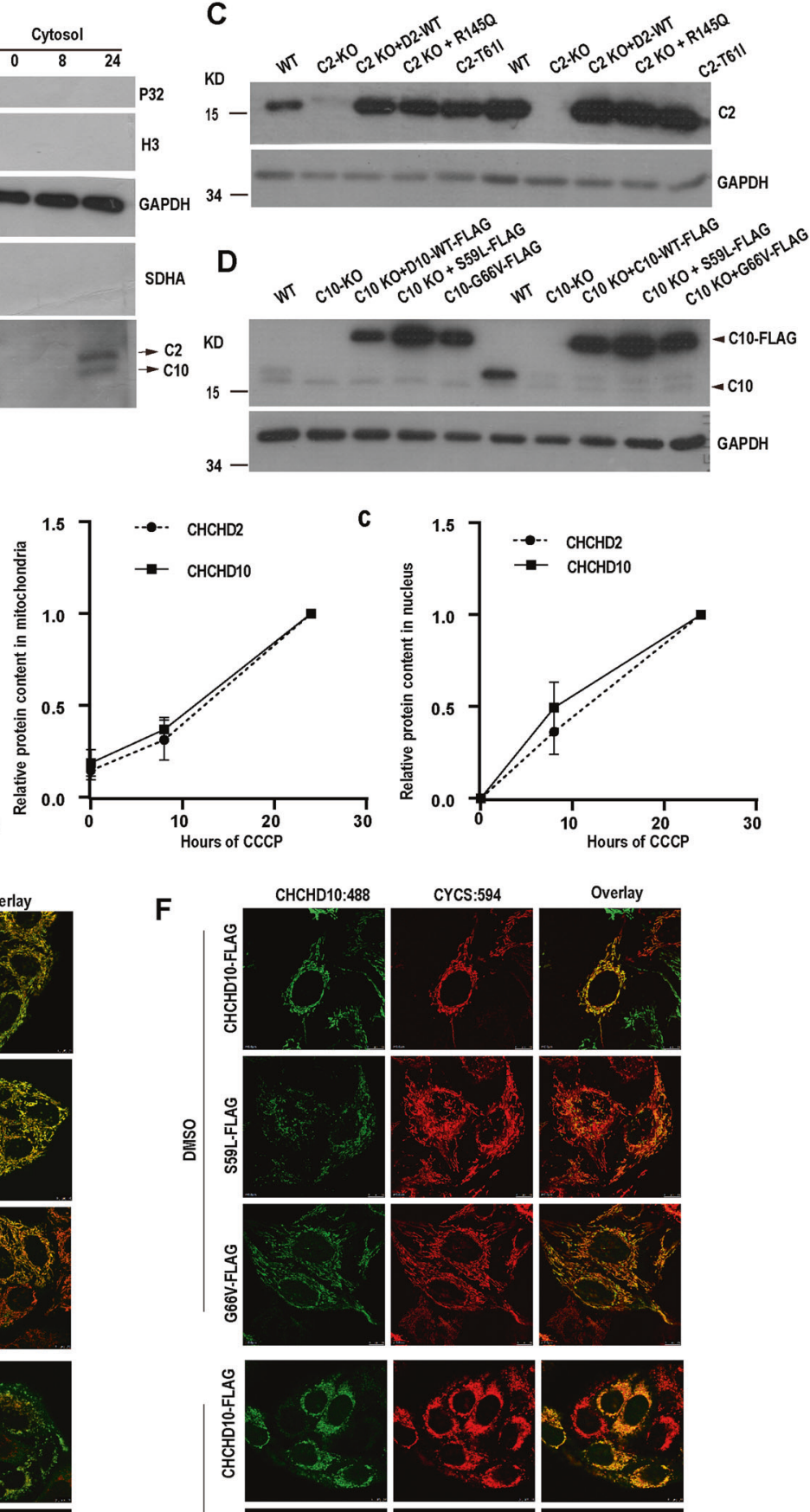

:
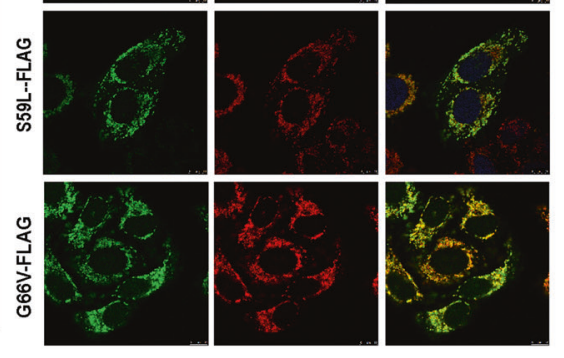

bind to OPA1 and DELE1 to inhibit its processing by protease; CHCHD2 and CHCHD10 bind OMA1 to suppress its activity. We observed that either $\mathrm{CHCHD} 2$ or $\mathrm{CHCHD} 10$ knockdown resulted in similar decrease of mitochondrial membrane potential and ATP production (Supplementary Fig. 2). However, CHCHD2 
Fig. 4 CCCP treatment increase CHCHD2 and CHCHD10 in mitochondria and cytosol. A Subcellular fractionation of HeLa cells treated with DMSO or CCCP $(10 \mathrm{mM}, 8 \mathrm{~h}$ or $24 \mathrm{~h})$ respectively were analyzed for indicated protein expression by immunoblot. B Quantitative analysis of $\mathrm{CHCHD} 2$ and $\mathrm{CHCHD} 10$ in nucleus (a), in mitochondria (b) and in cytosol (c) from A. The levels of CHCHD2 and CHCHD10 in nucleus, mitochondria, and cytosol are normalized to those of $\mathrm{H} 3, \mathrm{SDHA}$, and GAPDH respectively. $n=3$ independent samples, data are presented as mean values \pm SD. C Wild-type (WT) and clonal CHCHD2-knockout cells were transfected with indicated CDNA, treated with CCCP for $24 \mathrm{~h}$ and assayed by immunoblotting. D Wild-type (WT) and clonal CHCHD10-knockout cells, were transfected with indicated cDNA, and then treated with CCCP for $24 \mathrm{~h}$ and assayed by immunoblotting. E Subcellular localization of CHCHD2-WT, CHCHD2-T61l, or CHCHD2-R145Q in HeLa cells with or without CCCP treatment was analyzed by confocal microscopy. Mitochondria were visualized with CYCS staining. $\mathbf{F}$ Subcellular localization of FLAG-tagged CHCHD10 WT, CHCHD10-S59L, or CHCHD10-G66V in Hela Cells with or without CCCP treatment was analyzed by confocal microscopy. Mitochondria were visualized with Hsp60 staining.

knockdown, not CHCHD10, increased OMA1-mediated OPA1 cleavage in Hela cells (Fig. 1A). These data indicate that the lack of CHCHD2 activates OMA1 not because of bioenergetic collapse.

CHCHD2 and CHCHD10 could bind OMA1 but not OPA1 and DELE1 under normal conditions (Fig. 3C, E, Supplementary Fig. 3), so we speculated that $\mathrm{CHCHD} 2$ and $\mathrm{CHCHD} 10$ inhibitted the activity of OMA 1 by binding OMA1, but not by binding OPA 1 and DELE1 to suppress their processing under normal conditions.

CHCHD2 single knockdown leads to activation of OMA1, while CHCHD10 single knockdown does not lead to OMA1 activation, and $\mathrm{CHCHD} 2$ and $\mathrm{CHCHD} 10$ double knockdown lead to further activation of OMA1. Therefore, we hypothesized that $\mathrm{CHCHD} 2$ played a major role and $\mathrm{CHCHD} 10$ played a minor role in repressing the activity of OMA1 under normal circumstances. By immunoprecipitation, we observed that $\mathrm{CHCHD} 2$ and CHCHD10 also interacted elF2a under normal conditions, and surprisingly $\mathrm{CHCHD} 2$ and $\mathrm{CHCHD} 10$ interacted with elF2a more strongly under stress conditions than under normal conditions (Fig. 3D-F). In addition, more CHCHD2 and CHCHD10 entered to the cytosol under stress conditions (Fig. 4), and loss of CHCHD2 and $\mathrm{CHCHD} 10$ increased the phosphorylation of elF2a under normal conditions and stress conditions (Fig. 2B). Given that elF2 $a$ is phosphorylated during ISR initiation, therefore, we hypothesized that CHCHD2 and CHCHD10 interactted with elF2a to inhibit its phosphorylation, thereby regulating ISR under stress conditions.

Based on the above results, we propose a model: at normal conditions, $\mathrm{CHCHD} 2$ and $\mathrm{CHCHD} 10$ mainly suppress the OMA1 activity and inhibit phosphorylation of elF $2 a$ by binding them, while loss of CHCHD2 and CHCHD10 activate OMA1 to cleavage OPA1 and DELE1; at stress conditions (loss of membrane potential), CHCHD2 and CHCHD10 increase and translocate from mitochondria to the cytosol to suppress phosphorylation of elF2a to inhibit ISR (Fig. 7).

Finally, we discovered that P32, a partner of CHCHD2 and CHCHD10, can also regulate mitochondrial morphology and ISR. However, the detailed mechanisms are still to be investigated.

Mutations in $\mathrm{CHCHD} 2$ and $\mathrm{CHCHD} 10$ can lead to neurological diseases, while $\mathrm{CHCHD} 2$ and $\mathrm{CHCHD} 10$ are degraded by proteases under stress conditions. We find that Yme1l and OMA1 cooperate to degrade $\mathrm{CHCHD} 2$ and $\mathrm{CHCHD} 10$ under stress conditions (Fig. 6A), and the degradation rate of the PD-related mutations of $\mathrm{CHCHD2}$, CHCHD2-T61, and CHCHD2-R145Q is significantly slower than that of the wild-type CHCHD2 (Fig. 6B).

\section{MATERIALS AND METHODS \\ Cell culture}

HeLa (CCL-2, ATCC), 293 T (CRL-3216, ATCC), HCT116 (CCL-247, ATCC), and GP2-293(631512, TaKaRa) cells were cultured in Dulbecco's Modified Eagle Medium (DMEM) (11995065, Gibico) containing $4.5 \mathrm{gl}-1$ glucose, L-Glumine and sodium pyruvate supplemented with $10 \%$ fetal bovine serum (ST30-3302, PAN, Germany), and $1 \%$ sodium pyruvate at $37^{\circ} \mathrm{C}$ under $5 \% \mathrm{CO} 2$ conditions. All cell lines were tested for absence of mycoplasma contamination and authenticated using the short tandem repeat (STR) method.

\section{Stable cell generation}

Stable expression cell lines and rescued knockout cell lines were generated using retrovirus infections. Human CHCHD2 CDNA and its mutants were cloned into a modified pMSCV-puro (K1062-1, Clontech) vector whose puromycin resistance marker was deleted. Human DELE1 CDNA, CHCHD10 CDNA, CHCHD10-S59L, and CHCHD10-G66V cDNA with a C-terminal $3 \times$ Flag tag were also cloned into the modified pMSCV-puro vector whose puromycin resistance marker was deleted. Mouse OMA1 CDNA and OMA-E324Q were cloned into pMSCV-puro vector with a Cterminal $3 \times$ Flag tag. Retroviral particles were generated by transient transfection of GP2-293 cells seeded in 6-cm cell culture plates with VSV-G (\#8454, Addgene) and transfer plasmid using HighGene Transfection reagent (RM09014, ABclonal). Forty-eight hours later, the supernatant was collected and filtered, and then was added to cells. Cells were incubated with virus for $8 \mathrm{~h}$ with $10 \mu \mathrm{g} / \mathrm{ml}$ polybrene and then the medium was discarded and replaced with a fresh medium. One week after viral transduction, stable expression cell lines and rescued knockout cell lines were verified by Western Blot and immunoblotting.

CHCHD2 and CHCHD10 knockdown cell lines were generated using a modified retroviral vector as described previously [4]. P32 knockdown cell lines were generated using pLKO.1 puro (8453, Addgene). The following target sequences for gene knockdown were:

5'-CAGTGGAGGAAGTAATGCT-3' for CHCHD2;

5'-CCCTGAAGCAGTGCAAGTA-3' for CHCHD10;

5'-GGATGAGGTTGGACAAGAAGA-3' P32.

Retrovirus particles for shCHCHD2 and shCHCHD10 were generated as above. Lentivirus particles for shP32 were generated by transient transfection of 293 T cells seeded in $6-\mathrm{cm}$ cell culture plates with VSV-G (\#8454, Addgene), pCMV-dR8.2 dvpr(\#8455, Addgene), and lentiviral vector using HighGene Transfection reagent (RM09014, ABclonal), $48 \mathrm{~h}$ later, the supernatant was collected and filtered. Cells were incubated with virus for $8 \mathrm{~h}$ with $10 \mu \mathrm{g} / \mathrm{ml}$ polybrene and then the medium was discarded and replaced with fresh medium. Five days after infection, the loss of target protein expression was verified by immunoblotting.

Hela cells lacking CHCHD2 and CHCHD10, Hct116 cells lacking OMA1 or/ and Yme1L were generated by CRISPR/Cas9 gene editing, as described previously [48]. The following guide sequences were used:

5'-ACATTAGCATCCACCTCACG-3' for OMA1;

5'-TGTCCAAGTGTTGGCCCCCG-3' for Yme1L;

5'-CGGCCAGGTGAGACCATCGC-3' for CHCHD2;

5'-GAGATGGCGACCACGGCCGCA-3' for CHCHD10.

Designed Oligos were cloned into LentiCRISPR-V1 plasmid (49535, Addgene) and then were packaged into lentivirus in 293 T cells as above. At $48 \mathrm{~h}$ post-transfection, the supernatant containing lentiviral particles was collected and used for infecting cells with $10 \mu \mathrm{g} / \mathrm{ml}$ polybrene (H9268, Sigma-Aldrich). Eight hours later, the medium was discarded and replaced with a fresh medium. Twenty-four hours later, $2 \mu \mathrm{g} / \mathrm{ml}$ puromycin was added to the medium to select and establish stably transfected cells. Then, the single cells were sorted into 96-well dishes for the screen of knockout lines. The surviving clones were expanded, selected, and analyzed by immunoblotting. The genomic region flanking the targeting sequence was amplified by PCR and subjected to DNA sequencing.

\section{Reagents and antibodies}

Reagents used in this paper are listed as follows: Dimethyl sulfoxide (DMSO, 41639, Sigma-Aldrich); Carbonyl cyanide 3chlorophenylhydrazone (555-60-2, Sigma-Aldrich); Puromycin (A1113803, Invitrogen); Digitonin (D141, Sigma-Aldrich); Oligomycin (1404-19-9, Santa Cruz Biotechnology); and Opti-MEM (Thermo Fisher Scientific).

The following commercial antibodies were used for Western Blot: anti-Flag (Sigma-Aldrich, F1804, 1:5000 dilution); anti-OMA1, (Santa Cruz 
A
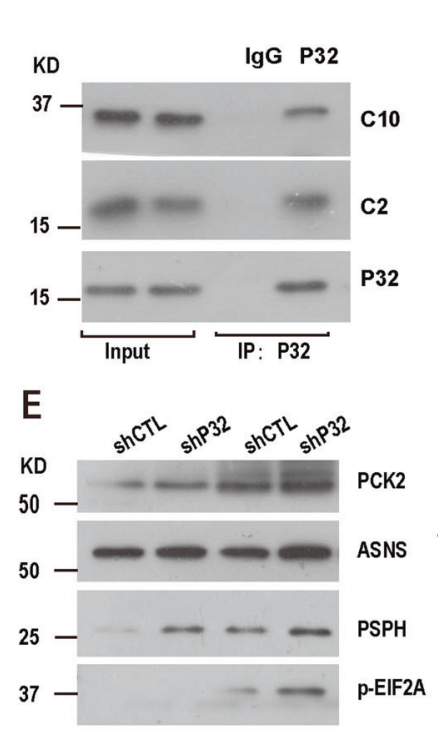

B

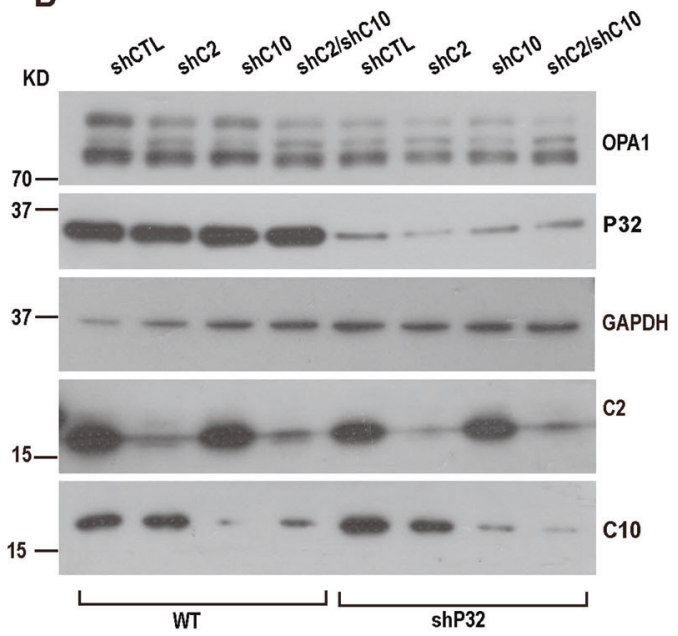

C
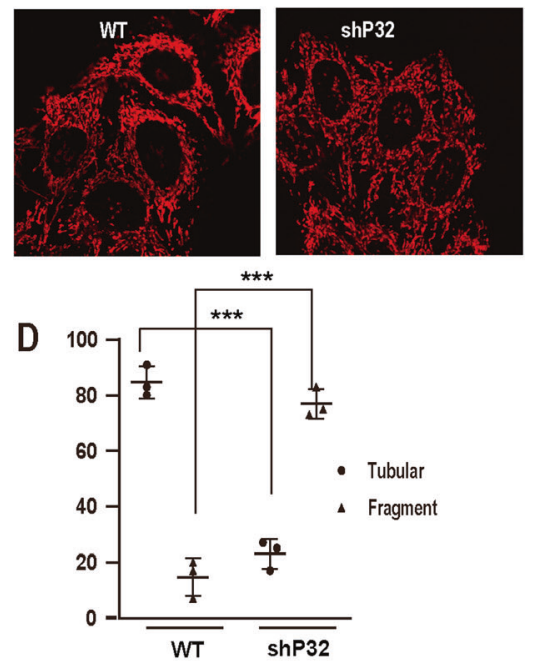

F
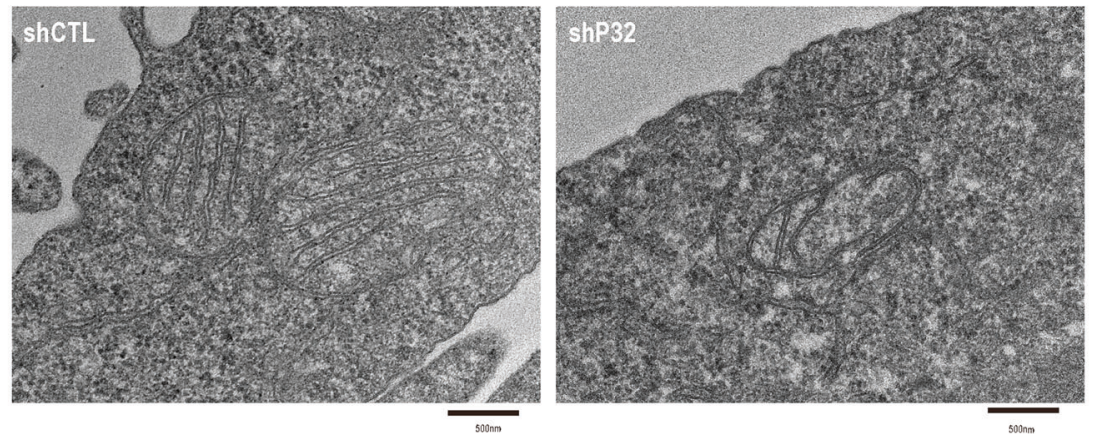

Fig. 5 P32 interacts with CHCHD2 and CHCHD10 to regulate mitochondrial morphology and mtISR. A Hela cell lysates were immunoprecipitated with anti-P32 antibody or lgG, followed by immunoblotting using the indicated antibodies. B Lysates of P32-knockdownHela cells treated with DMSO or CCCP $(10 \mathrm{uM}, 124 \mathrm{~h})$ analyzed for indicated protein expression by immunoblot. C Representative immunofluorescence images of mitochondrial morphology in control, shP32 Hela cells (mean \pm s.d. of $n=3$ independent biological samples; two-way ANOVA with Tukey's multiple comparisons correction, ${ }^{* * *} P<0.001$ ). D Mitochondrial morphology described in C was counted according to the criteria detailed in Experimental Procedures. E Representative western blots of the indicated proteins in WT and shP32 Hela cells after 10uM CCCP treatment for $24 \mathrm{~h}$. F Representative electron microscopic images and quantification of mitochondrial ultrastructure in WT or shP32 Hela cells.

Biotechnology, H-11, 1:200 dilution); anti-Yme1L (Proteintech, 11510-1-AP, 1:3000 dilution); anti-SDHA (Proteintech, 14865-1-AP, 1:3000 dilution); anti-OPA1 (BD, 612607, 1:2000 dilution), Anti-Drp1 (BD, 611738, 1:2000 dilution); Anti-ASNS (14681-1-AP, Proteintech, 1:1000 dilution); Anti-PCK2 (14892-1-AP, Proteintech, 1:1000 dilution); Anti-PSPH (Proteintech, 145131-AP, 1:1000 dilution); Anti-Fis1 (Proteintech, 10956-1-AP, 1:1000 dilution); Anti-Mff (Proteintech, 17090-1-AP, 1:1000 dilution); Anti-CHCHD2(Proteintech, 19424-1-AP, 1:1000 dilution); Anti-CHCHD10 (Proteintech, 25671-1AP, 1:1000 dilution); Anti-P32 (Abclonal, A1883, 1:5000 dilution); AntielF2a (Cell Signaling Technologies, \#5324, 1:3000 dilution); AntiPhosphorylated elF2a(Cell Signaling Technologies, \#3398, 1:1000 dilution), Anti-GAPDH (Cell Signaling Technologies, \#5174, 1:10000 dilution); Antiphosphorylated Drp1 (Ser616) (Cell Signaling Technologies, \#3455, 1:1000 dilution); Anti-phosphorylated Drp1 (Ser637) (Cell Signaling Technologies, \#4867, 1:1000 dilution); Anti-Actin (Sigma, A5441, 1:10000 dilution); Anti Hsp60 (Proteintech, 15282-1-AP, 1:5000 dilution); Anti-SDHA (Proteintech, 14865-1-AP, 1:5000 dilution).

\section{Immunostaining}

Cells grown on coverslips were fixed for 20 min with $4 \%$ paraformaldehyde at first, then washed three times with PBS, and permeabilized for $10 \mathrm{~min}$ with $0.1 \%$ Triton X-100 in PBS. After three washes with PBS, cells were blocked with $10 \%$ FBS in PBS for $1 \mathrm{~h}$ at room temperature, then incubated with primary antibody for $1 \mathrm{~h}$ at room temperature. After three washes with PBS, cells were incubated with secondary antibodies for $1 \mathrm{~h}$ at room temperature. Finally, cells were washed three times with PBS and then were analyzed by using a Leica confocal microscope.

\section{Confocal microscopy and image processing}

Fixed or living cells were visualized by confocal microscopy with a Leica Sp8 microscope with a 633 numerical aperture 1.35 oil objective [49]. To determine mitochondrial morphology, 100 cells were randomly selected for quantitative analysis and visually scored into three classifications (tubular, short tubular, fragmented). Tubular refers to cells with only tubular mitochondria. Tubular refers to cells in which greater than half of the mitochondrial mass existed as tubules as opposed to spherical fragments. Short tubular refers to cells in which less than half of the mitochondrial mass existed as tubules. In addition, all cells in this class contained at least three clearly tubular mitochondria. Finally, fragmented refers to cells that contain spherical mitochondrial fragments with no more than two short tubules found.

\section{Electron microscopy}

The procedure for transmission electron microscopy (TEM) was performed according to the previous report [50]. The $100 \mathrm{mM}$ sodium cacodylate buffer was replaced by $100 \mathrm{mM}$ phosphate buffer without $\mathrm{CaCl} 2$. The sections were supported on copper grids and then post-stained in uranyl acetate for $10 \mathrm{~min}$ and then in lead citrate for $15 \mathrm{~min}$, and the stained sections were imaged onto negatives using a JEM-1400 plus electron microscope operated at $100 \mathrm{kV}$. 

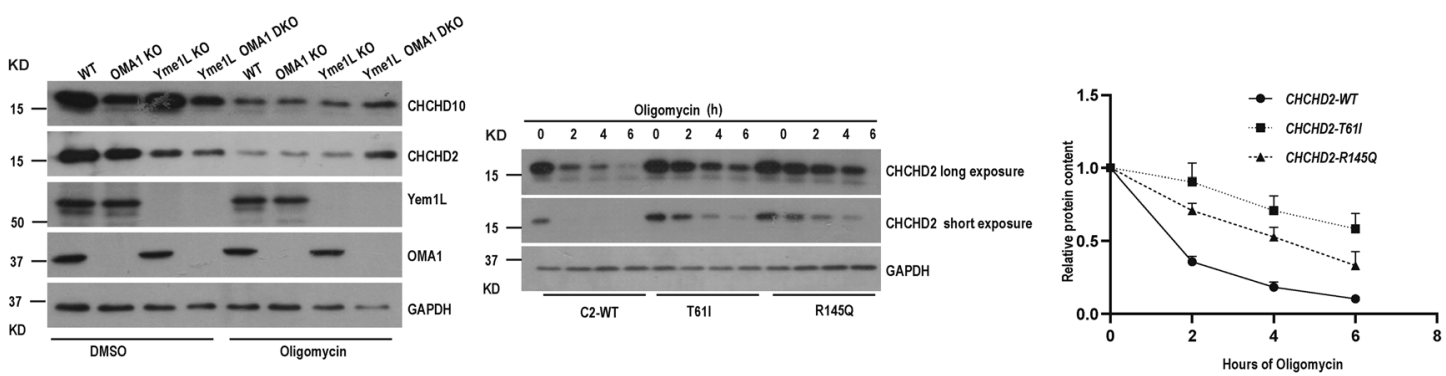

Fig. 6 Yme1L and OMA1 are responsible for degrading CHCHD2 and CHCHD10. A Immunoblot of OMA1 KO, Yme1L KO, OMA1 Yme1L DKO, and WT HeLa cells treated with dimethyl sulfoxide (DMSO) or $10 \mu \mathrm{m}$ Oligomycin for $8 \mathrm{~h}$. GAPDH served as loading controls. B Immunoblot of WT and CHCHD2 KO HeLa cell lines which stably express CHCHD2 WT, T61I or R145Q mutation where indicated, treated with either vehicle or $125 \mu \mathrm{m}$ Oligomycin for $6 \mathrm{~h}$. GAPDH served as the loading control. C Quantification of levels CHCHD2 and its mutation CHCHD2-T61l and CHCHD2-R145Q from B. The relative protein levels were evaluated by densitometry analysis using ImageJ software and were quantified for the ratio of $\mathrm{CHCHD2/GAPDH,} \mathrm{T61I} \mathrm{/GAPDH,} \mathrm{and} \mathrm{R145Q/GAPDH} \mathrm{(} n=3$ independent experiments). The data are presented as mean \pm SD.

\section{Quantitative real-time PCR analysis}

RNA from cells or tissues was extracted using RNAiso Plus reagent (TaKaRa Biotechnology, 9109) following the manufacturer's protocol. And the CDNA was synthesized using the ABScript II cDNA First Strand Synthesis Kit (ABclonal, RK20400) according to the manufacturer's instructions. Then, the CDNA samples were used as templates for quantitative real-time quantitative PCR analysis by using an SYBR Green Select Master Mix (ABclonal, RK21203) and the iCycler real-time PCR Detection System (BioRad). The fold change of target mRNA expression was calculated using the $2^{-\triangle \Delta C T}$ method. The $\beta$-ACTIN or GAPDH housekeeping gene was used for normalization. The primers used in this study were as followed:

CHCHD2: forward, 5'-GTGGAGGAAGTAATGCTGAGCC-3', and reverse, 5'CACAGAGCTTGATGTCACCCTG-3', CHCHD10: forward, 5'-ATCTGGTGTTGTG GTCTGGCTG-3', and reverse, 5'-GTGAGTGAGTGGACCCCGAC-3', ATF3: forward, 5'-GGAGCCTGGAGCAAAATGATG-3', and reverse, $5^{\prime}$-AGGGCG TCAGGTTAGCAAAA-3', CHAC1: forward, 5'-GTGGTGACGCTCCTTGAAGA-3', and forward, 5'-TTCAGGGCCTTGCTTACCTG-3', DDIT3: forward, 5'-AGC CAAAATCAGAGCTGGAA-3', and forward, 5'-TGGATCAGTCTGGAAAAGC A-3', ATF4: forward, 5'-CAGCAAGGAGGATGCCTTCT- $3^{\prime}$, and forward, 5'-CCA ACAGGGCATCCAAGTC- $3^{\prime}$, $\beta$-ACTIN: forward, 5 '-GGCATGGGTCAGAAGGAT $\mathrm{T}-3^{\prime}$, and reverse, $5^{\prime}$-CCACACGCAGCTCATTGTA-3'.

\section{Subcellular fractionation}

Cell fractionation and mitochondria isolation were performed as previously reported [51]. Briefly, following the $8 \mathrm{~h}$ or $24 \mathrm{~h} \mathrm{CCCP} \mathrm{treatment,}$ cells were collected and centrifuged at $400 \times \mathrm{g}$ for $5 \mathrm{~min}$ at $4{ }^{\circ} \mathrm{C}$. The cell pellet was washed once with PBS and permeabilized with $10 \mathrm{mg} / \mathrm{ml}$ digitonin (Sigma, D141) in PBS for $10 \mathrm{~min}$ at room temperature. Cells were centrifuged at $10,000 \times \mathrm{g}$ for $10 \mathrm{~min}$ at $4^{\circ} \mathrm{C}$, and supernatants were collected to a fresh tube (cytosolic fraction). The pellets were resuspended in the mitochondrial lysate buffer $(10 \mathrm{mM}$ Tris- $\mathrm{HCl} \mathrm{pH} \mathrm{7.4,150} \mathrm{mM} \mathrm{NaCl}$, $2 \mathrm{mM}$ EDTA, $0.2 \%$ Triton, $0.3 \%$ NP40, protease inhibitor cocktails) for $30 \mathrm{~min}$ on ice and centrifuged at $10,000 \times \mathrm{g}$ for $20 \mathrm{~min}$ at $4^{\circ} \mathrm{C}$. The supernatants and the pellets were the mitochondrial fraction and nuclear fraction respectively.

\section{Western blotting and co-immunoprecipitations}

Western blotting and co-immunoprecipitation (co-IP) analyses were performed as previously described. In brief, cells were lysed with RIPA buffer (Beyotime, P0013B) and complete protease inhibitor (Roche). The proteins were loaded onto an SDS-polyacrylamide gel, separated by electrophoresis, and blotted onto a PVDF membrane (Merck Millipore). For the co-IP, all steps were performed at $4{ }^{\circ} \mathrm{C}$, cells were solubilized with IP buffer $(20 \mathrm{mM}$ Tris- $\mathrm{HCl} \mathrm{pH}=7.4,150 \mathrm{mM} \mathrm{NaCl}, 2 \mathrm{mM}$ EDTA, $1 \%$ Triton X100 and protease inhibitor mixture) for $1 \mathrm{~h}$. The lysates were centrifuged at $12,000 \times g$ for $15 \mathrm{~min}$ and the supernatant was subsequently incubated with anti-CHCHD2 antibody and protein $\mathrm{G}$ conjugated magnetic beads (Bio-Rad, G-1614823) or anti-Flag M2 affinity gel (Sigma-Aldrich, A2220) at $4{ }^{\circ} \mathrm{C}$ overnight. The affinity gel was washed five times with lysis buffer, and then, the proteins were recovered by boiling the beads in sample buffer and analyzed by Western blotting.

\section{ROS measurement}

ROS levels were measured using MitoSox (M36008, Thermo Fisher Scientific). In brief, cells were stained with $5 \mu \mathrm{M}$ MitoSox at $37^{\circ} \mathrm{C}$ for 30 min and then analyzed by Cyto FLEX LX (Beckman Coulter). CCCP was used as a control of the technique.

\section{Membrane potential measurement}

Mitochondrial membrane potential $(\Delta \psi \mathrm{m})$ was measured using TMRM (M20036, Thermo Fisher Scientific). In brief, cells were stained with $200 \mathrm{nM}$ TMRM at $37^{\circ} \mathrm{C}$ for $15 \mathrm{~min}$ and then analyzed by Cyto FLEX LX (Beckman Coulter). CCCP was used as a control of the technique.

\section{ATP measurement}

Cellular ATP levels were measured using an ATP assay kit (Celltiter-Glo Luminescent Cell Viability Assay, G7573, Promega) according to the manufacturer's instructions. Briefly, cells were planted in 96-well plates and cells were cultured in DMEM without glucose $(11966025$, Gibco) containing L-glutamine supplemented with $10 \mathrm{mM}$ galactose (G5388, Sigma), $100 \mu \mathrm{M}$ non-essential amino acids, $1 \mathrm{mM}$ sodium pyruvate, and 10\% FBS (ST303302 , PAN). Whole-cell lysates were generated using the luciferase reporter lysis buffer. Luminescence was measured using the microplate reader and the values were normalized to the protein concentration. CCCP was used as a control of the technique.

\section{Cell proliferation assay}

Cell proliferation was measured using a Cell Counting Kit (CCK-8, C0042, Beyontime) according to the manufacturer's instructions. The absorbance of control and knockout cells was measured with the microplate reader at $450 \mathrm{~nm}$.

\section{Seahorse}

Oxygen consumption rates (OCR) of cells were measured with a Seahorse Extracellular Flux Analyzer XF96 (Agilent), according to the manufacturer's instructions. In brief, Hela cells were seeded in an XF96-well plate at a density of $1.5 \times 10^{4}$ per well and allowed to attach overnight. The standard 'mitochondria stress test' was performed consisting of basal measurements followed by measurements after sequential addition of $1 \mu \mathrm{m}$ oligomycin, $1.5 \mu \mathrm{m}$ FCCP, and $1 \mu \mathrm{m}$ of rotenone and antimycin. Data are presented as means \pm standard error of the mean for five replicates. Protein concentrations determined by BCA assay were used to normalize the OCRs.

\section{Photoactivatable GFP assay}

Mitochondrial targeted DesRed (mito-DsRed) and photoactivatable GFP (PA-GFP) targeted to the mitochondrial matrix (mito-PA-GFP) were stably expressed in cells and performed PA-GFP assay. Within a single cell, a small subset of mitochondria was photoactivated by excitation at $405 \mathrm{~nm}$, and then the mitochondrial fusion and fission events were tracked by timelapse microscopy for about $20 \mathrm{~min}$. 


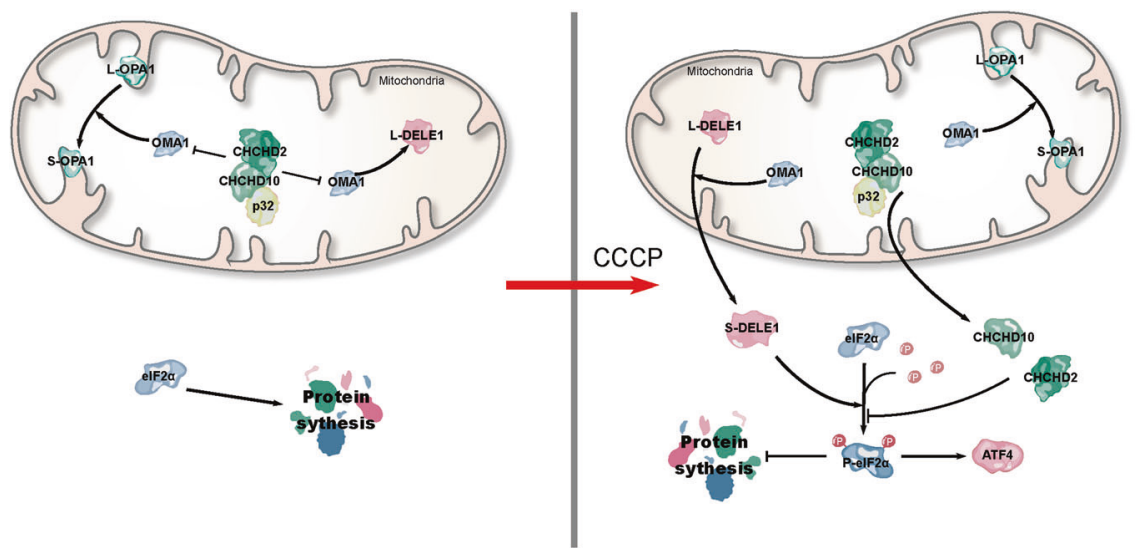

Fig. 7 Model of CHCHD2 and CHCHD10 regulating OMA1 activity and mtISR. CHCHD2 and CHCHD10 mainly suppress the OMA1 activity and inhibit phosphorylation of elF2A at normal conditions by binding them, and loss of CHCHD2 and CHCHD10 activates OMA1 to cleavage OPA1 and DELE1; at stress conditions (loss of membrane potential), CHCHD2 and CHCHD10 increase and translocate from mitochondria to cytosol to suppress phosphorylation of elF2A to inhibit ISR.

\section{Statistical analyses}

All statistical analyses were performed using Excel (Microsoft) or Prism (Graphpad 8.0). All statistical results are presented as the mean \pm standard deviation (S.D.), and $p$-values were calculated using two-tailed Student's $t$-test for pairwise comparisons, one-way ANOVA with Dunnett's multiple comparisons test for multiple comparisons, and two-way ANOVA with Tukey's multiple comparisons test for multiple comparisons test involving two independent variables. $p$-values $<0.05$ were considered significant. In all experiments, variances between groups were not statistically different.

\section{Statistics and reproducibility}

All experiments were performed independently at least three times unless stated otherwise in the figure legend. The sample size was chosen in accordance with the general standards and prior publications in the respective fields $[37,52]$. No statistical method was used to predetermine the sample size. No data were excluded from the analyses. The experiments were not randomized. The investigators were not blinded to allocation during experiments and outcome assessment.

\section{DATA AVAILABILITY}

The data generated or analyzed are included in this article and its supplementary files.

\section{REFERENCES}

1. Mishra $P, C h a n D C$. Mitochondrial dynamics and inheritance during cell division, development and disease. Nat Rev Mol Cell Biol. 2014;15:634-46.

2. Loson OC, Song Z, Chen H, Chan DC. Fis1, Mff, MiD49, and MiD51 mediate Drp1 recruitment in mitochondrial fission. Mol Biol Cell 2013;24:659-67.

3. Song Z, Ghochani M, McCaffery JM, Frey TG, Chan DC. Mitofusins and OPA1 mediate sequential steps in mitochondrial membrane fusion. Mol Biol Cell 2009;20:3525-32.

4. Song Z, Chen H, Fiket M, Alexander C, Chan DC. OPA1 processing controls mitochondrial fusion and is regulated by mRNA splicing, membrane potential, and Yme1L. J Cell Biol 2007;178:749-55.

5. Hu C, Shu L, Huang X, Yu J, Li L, Gong L, et al. OPA1 and MICOS Regulate mitochondrial crista dynamics and formation. Cell Death Dis 2020;11:940.

6. Frezza C, Cipolat S, Martins de Brito O, Micaroni M, Beznoussenko GV, Rudka T, et al. OPA 1 controls apoptotic cristae remodeling independently from mitochondrial fusion. Cell 2006;126:177-89.

7. Malpass K. Neurodegenerative disease: defective mitochondrial dynamics in the hot seat-a therapeutic target common to many neurological disorders? Nat Rev Neurol. 2013;9:417.

8. Chen $\mathrm{H}$, Chan DC. Mitochondrial dynamics-fusion, fission, movement, and mitophagy-in neurodegenerative diseases. Hum Mol Genet. 2009;18:R169-176.

9. Chen $\mathrm{H}$, Chan DC. Mitochondrial dynamics in mammals. Curr Top Dev Biol 2004;59:119-44.

10. Guo X, Aviles G, Liu Y, Tian R, Unger BA, Lin YT, et al. Mitochondrial stress is relayed to the cytosol by an OMA1-DELE1-HRI pathway. Nature 2020;579:427-32.
11. Fessler E, Eckl EM, Schmitt S, Mancilla IA, Meyer-Bender MF, Hanf M, et al. A pathway coordinated by DELE1 relays mitochondrial stress to the cytosol. Nature 2020;579:433-7.

12. Quiros PM, Prado MA, Zamboni N, D'Amico D, Williams RW, Finley D, et al. Multiomics analysis identifies ATF4 as a key regulator of the mitochondrial stress response in mammals. J Cell Biol. 2017;216:2027-45.

13. Funayama M, Ohe K, Amo T, Furuya N, Yamaguchi J, Saiki S, et al. CHCHD2 mutations in autosomal dominant late-onset Parkinson's disease: a genome-wide linkage and sequencing study. Lancet Neurol. 2015;14:274-82.

14. Bannwarth S, Ait-El-Mkadem S, Chaussenot A, Genin EC, Lacas-Gervais S, Fragaki $\mathrm{K}$, et al. A mitochondrial origin for frontotemporal dementia and amyotrophic lateral sclerosis through CHCHD10 involvement. Brain 2014;137:2329-45.

15. Muller K, Andersen PM, Hubers A, Marroquin N, Volk AE, Danzer KM, et al. Two novel mutations in conserved codons indicate that CHCHD10 is a gene associated with motor neuron disease. Brain 2014;137:e309.

16. Chaussenot A, Le Ber I, Ait-El-Mkadem S, Camuzat A, de Septenville A, Bannwarth $S$, et al. Screening of CHCHD10 in a French cohort confirms the involvement of this gene in frontotemporal dementia with amyotrophic lateral sclerosis patients. Neurobiol Aging. 2014;35:2884 e2881-2884 e2884.

17. Kurzwelly D, Kruger S, Biskup S, Heneka MT. A distinct clinical phenotype in a German kindred with motor neuron disease carrying a $\mathrm{CHCHD} 10$ mutation. Brain: a J Neurol. 2015;138:e376.

18. Perrone F, Nguyen HP, Van Mossevelde S, Moisse M, Sieben A, Santens $P$, et al. Investigating the role of ALS genes CHCHD10 and TUBA4A in Belgian FTD-ALS spectrum patients. Neurobiol Aging. 2017;51:177 e179-177 e116.

19. Auranen M, Ylikallio E, Shcherbii M, Paetau A, Kiuru-Enari S, Toppila JP, et al. CHCHD10 variant p.(Gly66Val) causes axonal Charcot-Marie-Tooth disease. Neurol Genet. 2015;1:e1.

20. Pasanen P, Myllykangas L, Poyhonen M, Kiuru-Enari S, Tienari PJ, Laaksovirta $H_{\text {, }}$ et al. Intrafamilial clinical variability in individuals carrying the $\mathrm{CHCHD} 10$ mutation Gly66Val. Acta Neurol Scand. 2016;133:361-6.

21. Penttila S, Jokela M, Saukkonen AM, Toivanen J, Palmio J, Lahdesmaki J, et al CHCHD10 mutations and motor neuron disease: the distribution in Finnish patients. J Neurol Neurosurg Psychiatry 2017;88:272-7.

22. Ajroud-Driss S, Fecto F, Ajroud K, Lalani I, Calvo SE, Mootha VK, et al. Mutation in the novel nuclear-encoded mitochondrial protein $\mathrm{CHCHD10}$ in a family with autosomal dominant mitochondrial myopathy. Neurogenetics 2015;16:1-9.

23. Imai Y, Meng HR, Shiba-Fukushima K, Hattori N. Twin $\mathrm{CHCH}$ Proteins, CHCHD2, and CHCHD10: key molecules of Parkinson's disease, amyotrophic lateral sclerosis, and frontotemporal dementia. Int J Mol Sci. 2019;20:908.

24. Burstein SR, Valsecchi F, Kawamata $H$, Bourens $M$, Zeng $R$, Zuberi $A$, et al. In vitro and in vivo studies of the ALS-FTLD protein CHCHD10 reveal novel mitochondrial topology and protein interactions. Hum Mol Genet. 2018;27:160-77.

25. Huang X, Wu BP, Nguyen D, Liu YT, Marani M, Hench J, et al. CHCHD2 accumulates in distressed mitochondria and facilitates oligomerization of CHCHD10. Hum Mol Genet. 2019;28:349.

26. Straub IR, Janer A, Weraarpachai W, Zinman L, Robertson J, Rogaeva E, et al. Loss of $\mathrm{CHCHD} 10-\mathrm{CHCHD} 2$ complexes required for respiration underlies the pathogenicity of a CHCHD10 mutation in ALS. Hum Mol Genet. 2018;27:178-89. 
27. Aras S, Pak O, Sommer N, Finley R Jr., Huttemann M, Weissmann N, et al. Oxygendependent expression of cytochrome $c$ oxidase subunit 4-2 gene expression is mediated by transcription factors RBPJ, CXXC5 and CHCHD2. Nucleic Acids Res. 2013;41:2255-66.

28. Grossman LI, Purandare N, Arshad R, Gladyck S, Somayajulu M, Huttemann M, et al. MNRR1, a biorganellar regulator of mitochondria. Oxid Med Cell Longev. 2017;2017:6739236.

29. Liu Y, Clegg HV, Leslie PL, Di J, Tollini LA, He Y, et al. CHCHD2 inhibits apoptosis by interacting with $\mathrm{BCl}-\mathrm{x} \mathrm{L}$ to regulate Bax activation. Cell Death Differ. 2015;22:1035-46.

30. Sato S, Noda S, Torii S, Amo T, Ikeda A, Funayama M, et al. Homeostatic p62 levels and inclusion body formation in $\mathrm{CHCHD} 2$ knockout mice. Human molecular genetics. 2021.

31. Purandare N, Somayajulu M, Huttemann M, Grossman LI, Aras S. The cellular stress proteins CHCHD10 and MNRR1 (CHCHD2): partners in mitochondrial and nuclear function and dysfunction. J Biol Chem 2018;293:6517-29.

32. Genin EC, Bannwarth S, Lespinasse F, Ortega-Vila B, Fragaki K, Itoh K, et al. Loss of MICOS complex integrity and mitochondrial damage, but not TDP-43 mitochondrial localisation, are likely associated with severity of CHCHD10-related diseases. Neurobiol Dis 2018;119:159-71.

33. Genin EC, Plutino $M$, Bannwarth $S$, Villa $E$, Cisneros-Barroso $E$, Roy $M$, et al. CHCHD10 mutations promote loss of mitochondrial cristae junctions with impaired mitochondrial genome maintenance and inhibition of apoptosis. EMBO Mol Med. 2016;8:58-72.

34. Zhou W, Ma D, Sun AX, Tran HD, Ma DL, Singh BK, et al. PD-linked CHCHD2 mutations impair CHCHD10 and MICOS complex leading to mitochondria dysfunction. Hum Mol Genet. 2019;28:1100-16.

35. Liu YT, Huang X, Nguyen D, Shammas MK, Wu BP, Dombi E, et al. Loss of CHCHD2 and $\mathrm{CHCHD} 10$ activates OMA1 peptidase to disrupt mitochondrial cristae phenocopying patient mutations. Hum Mol Genet. 2020;29:1547-67.

36. Anderson CJ, Bredvik K, Burstein SR, Davis C, Meadows SM, Dash J, et al. ALS/FTD mutant $\mathrm{CHCHD} 10$ mice reveal a tissue-specific toxic gain-of-function and mitochondrial stress response. Acta Neuropathologica. 2019;138:103-21.

37. Ruan Y, Li H, Zhang K, Jian F, Tang J, Song Z. Loss of Yme1L perturbates mitochondrial dynamics. Cell Death Dis. 2013;4:e896.

38. Kondadi AK, Anand R, Reichert AS. Cristae Membrane Dynamics - A Paradigm Change. Trends Cell Biol. 2020;30:923-36.

39. Huang X, Wu BP, Nguyen D, Liu YT, Marani M, Hench J, et al. CHCHD2 accumulates in distressed mitochondria and facilitates oligomerization of CHCHD10. Hum Mol Genet. 2018;27:3881-3900.

40. Narendra $D$, Liu $Y T$, Huang $X P$, Nguyen $D$, Shammas $M, W u$, et al. Loss of CHCHD2 and CHCHD10 Activates Oma1 Peptidase to Disrupt Mitochondrial Cristae Phenocopying Patient Mutations. Ann Neurol. 2020;88:S215-S215.

41. Liu YT, Huang XP, Nguyen D, Shammas MK, Wu BP, Dombi E, et al. Loss of CHCHD2 and CHCHD10 activates OMA1 peptidase to disrupt mitochondrial cristae phenocopying patient mutations. Hum Mol Genet. 2020;29:1547-67.

42. Huang XP, Wu BP, Nguyen D, Liu YT, Marani M, Hench J, et al. CHCHD2 accumulates in distressed mitochondria and facilitates oligomerization of $\mathrm{CHCHD} 10$. Hum Mol Genet. 2018;27:3881-3900.

43. Fogal V, Richardson AD, Karmali PP, Scheffler IE, Smith JW, Ruoslahti E. Mitochondrial p32 protein is a critical regulator of tumor metabolism via maintenance of oxidative phosphorylation. Mol Cell Biol 2010;30:1303-18.

44. Li Y, Wan OW, Xie W, Chung KK. p32 regulates mitochondrial morphology and dynamics through parkin. Neuroscience 2011;199:346-58.

45. Noh $\mathrm{S}$, Phorl $\mathrm{S}$, Naskar $\mathrm{R}$, Oeum $\mathrm{K}$, Seo $\mathrm{Y}$, Kim $\mathrm{E}$, et al, p32/C1OBP regulates OMA1dependent proteolytic processing of OPA1 to maintain mitochondrial connectivity related to mitochondrial dysfunction and apoptosis. Sci Rep. 2020;10:10618.

46. Liu W, Duan XY, Xu LN, Shang WN, Zhao JY, Wang LQ, et al. Chchd2 regulates mitochondrial morphology by modulating the levels of Opa1. Cell Death Differ. 2020;27:2014-29.

47. Alavi MV. OMA1-An integral membrane protease? Biochim Biophys Acta Proteins Proteom. 2021;1869:140558.
48. Wu Z, Zuo M, Zeng L, Cui K, Liu B, Yan C, et al. OMA1 reprograms metabolism under hypoxia to promote colorectal cancer development. EMBO Rep. 2021;22: e50827.

49. Correction for Aras et al., Mitochondrial Nuclear Retrograde Regulator 1 (MNRR1) rescues the cellular phenotype of MELAS by inducing homeostatic mechanisms. Proc Natl Acad Sci USA. 2021;118.

50. Jian F, Chen D, Chen L, Yan C, Lu B, Zhu Y, et al. Sam50 regulates PINK1-ParkinMediated mitophagy by controlling PINK1 Stability and mitochondrial morphology. Cell Rep. 2018;23:2989-3005.

51. Baghirova S, Hughes BG, Hendzel MJ, Schulz R. Sequential fractionation and isolation of subcellular proteins from tissue or cultured cells. MethodsX 2015;2:440-5.

52. Tang J, Zhang K, Dong J, Yan C, Hu C, Ji H, et al. Sam50-Mic19-Mic60 axis determines mitochondrial cristae architecture by mediating mitochondrial outer and inner membrane contact. Cell Death Differ. 2020;27:146-60.

\section{ACKNOWLEDGEMENTS}

We thank Dr. Zhida Wu for the support with fluorescence microscopy and electron microscopy. This work was financially supported by the National Key R\&D Program of China (2021YFA0804900) and the 111 Project (B16021).

\section{AUTHOR CONTRIBUTIONS}

Y.R. designed and performed most of the experiments and analyzed the data; Y.C., J.H., Y.L., Z.L., and J.C. performed experiments; Q.H. revised the manuscript; H.H. and Q.Z. conceived and directed the project; Y.R. and Q.Z. wrote the manuscript.

\section{COMPETING INTERESTS}

The authors declare no competing interests.

\section{ADDITIONAL INFORMATION}

Supplementary information The online version contains supplementary material available at https://doi.org/10.1038/s41419-022-04602-5.

Correspondence and requests for materials should be addressed to $\mathrm{He} \mathrm{He}$ or Qinghua Zhou.

Reprints and permission information is available at http://www.nature.com/ reprints

Publisher's note Springer Nature remains neutral with regard to jurisdictional claims in published maps and institutional affiliations.

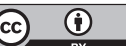

Open Access This article is licensed under a Creative Commons Attribution 4.0 International License, which permits use, sharing, adaptation, distribution and reproduction in any medium or format, as long as you give appropriate credit to the original author(s) and the source, provide a link to the Creative Commons license, and indicate if changes were made. The images or other third party material in this article are included in the article's Creative Commons license, unless indicated otherwise in a credit line to the material. If material is not included in the article's Creative Commons license and your intended use is not permitted by statutory regulation or exceeds the permitted use, you will need to obtain permission directly from the copyright holder. To view a copy of this license, visit http://creativecommons. org/licenses/by/4.0/

(c) The Author(s) 2022 